\title{
Fluctuation splitting Riemann solver for a non-conservative modeling of shear shallow water flow.
}

\author{
Ashish Bhole*1, Boniface Nkonga ${ }^{\dagger 1}$, Sergey Gavrilyuk ${ }^{\ddagger 2}$, and Kseniya Ivanova ${ }^{\S 2}$ \\ ${ }^{1}$ Université Côte d'Azur (UCA), JAD/C.N.R.S. U.M.R. 7351, Parc Valrose, \\ 06108 NICE Cedex 2, France \\ 22Aix-Marseille Université, C.N.R.S. U.M.R. 7343, IUSTI, 5 rue E. Fermi, 13453 \\ Marseille Cedex 13 France
}

\begin{abstract}
In this paper we propose a fluctuation splitting finite volume scheme for a non-conservative modeling of shear shallow water flow (SSWF). This model was originally proposed by Teshukov (2007) in [14] and was extended to include modeling of friction by Gavrilyuk et al. (2018) in [7]. The directional splitting scheme proposed by Gavrilyuk et al. (2018) in [7] is tricky to apply on unstructured grids. Our scheme is based on the physical splitting in which we separate the characteristic waves of the model to form two different hyperbolic sub-systems. The fluctuations associated with each sub-systems are computed by developing Riemann solvers for these subsystems in a local coordinate system. These fluctuations enables us to develop a Godunov-type scheme that can be easily applied on mixed/unstructured grids. While the equation of energy conservation is solved along with the SSWF model in Gavrilyuk et al. (2018)[7], in this paper we solve only SSWF model equations.

We develop a cell-centered finite volume code to validate the proposed scheme with the help of some numerical tests. As expected, the scheme shows first order convergence. The numerical simulation of $1 \mathrm{D}$ roll waves shows a good agreement with the experimental results. The numerical simulations of 2D roll waves show similar transverse wave structures as observed by Gavrilyuk et al. (2018) in [7].
\end{abstract}

\section{Keywords}

Shear shallow water flows; Finite volume methods; Unstructured grids; Riemann solvers; Nonconservative hyperbolic system; Family of paths

\section{Introduction}

In the present paper we investigate nonlinear, non-conservative hyperbolic models arising in the modeling of shear shallow water flows (SSWF). The main challenge for the numerical strategies for a non-conservative hyperbolic systems lies in the fact that the jump conditions across shocks

\footnotetext{
*ashish.bhole@unice.fr

†boniface.nkonga@unice.fr

‡sergey.gavrilyuk@univ-amu.fr

§ivanova.kseniya15@gmail.com
} 
are not valid. The presence of non-conservative products questions the the concept of weak solutions. In [11] Dal Maso, LeFloch and Murat proposed a definition of the non-conservative products as Borel measures based on a choice of a "family of paths" in the phase space. This allows to clarify the concept of weak solution. Different weak solutions will be obtained with different "family of paths". Therefore we have to define a preferable "family of paths" based on some physical principles. Entropy inequality can help us to choose the "family of paths" with the admissible speed of shock propagation. In this framework, the theory of simple waves and the resolution of the Riemann problem can be extended to non-conservative hyperbolic systems. The associated notion of Riemann solver is the building block of the so called Godunov-type schemes $[8,12]$ for numerical approximation of conservative or non-conservative hyperbolic systems.

In [2] various flux vector splittings for the magneto-hydrodynamic system are explored. In [6] Dumbser and Balsara provides a derivation of the HLL Riemann solver for non-conservative systems. This strategy is based on the Heaviside weights. In [1] a simplified version based on the parametrization of the Riemann solver along a preferable path is proposed. This article emphasizes the need to take into account the characteristics of the physical model, in the design of the "family of paths". In this context, the HLL Riemann solver is extended to a class of non-conservative hyperbolic systems. For instance, the intermediate state is implicitly defined by a nonlinear system which can be solved by the Newton's procedure.

The SSWF model with the flat bottom is proposed in $[14,7]$. The characteristic analysis of SSWF is studied and the numerical scheme based on the directional splitting is proposed in [7]. The directional splitting strategy makes the numerical scheme unsuitable for unstructured grids. In this paper we propose a fluctuation splitting strategy that can be applied on mixed unstructured grids. The splitting is physical in which one separates the characteristic waves in two parts each corresponding to a hyperbolic sub-system. Riemann solvers are developed for these hyperbolic sub-systems in a local coordinate system. These Riemann solvers allow us to compute the fluctuations associated with each sub-system and write a Godunov-type scheme for SSWF model. Such fluctuation formulation of the Riemann solver, particularly in the context of non-conservative systems, is convenient for the splitting techniques. The proposed approach fits into the extensions of so called TV flux splitting $[17,18,15]$. The resulting method is addressed for accuracy on an analytic solution and for robustness on a carefully selected problems. Unlike [7], where the equation of energy conservation is solved along with the SSWF model, in this paper we solve only SSWF model equation.

In Section 2 the dissipative SSWF model is described with the focus on the dissipative source terms. In Section 3 we describe the non-dissipative part of the model, physical splitting of the model, associated Riemann problems and the fluctuation splitting scheme. The numerical strategy for one and multi-dimensional problems is presented in Section 4. Some 1D and 2D numerical tests are presented in Section 5 to validate the proposed scheme. Section 6 outlines the conclusions.

\section{Dissipative model}

The model for SSWF has been recently proposed in [7]. Mathematically it is a system of nonlinear, non-conservative hyperbolic partial differential equations (PDEs) describing the evolution of the depth $(h)$, the depth average momentum $(h \boldsymbol{u})$ and the stress tensor $(\mathbb{P})$.

$$
\left\{\begin{array}{lll}
\partial_{t} h & +\nabla \cdot(h \boldsymbol{u}) & =0 \\
\partial_{t}(h \boldsymbol{u}) & +\nabla \cdot(h \boldsymbol{u} \otimes \boldsymbol{u}+p \mathbf{I}+h \mathbb{P}) & =-g h \nabla \mathrm{b}-C_{f}|\mathbf{u}| \mathbf{u} \text { where } p=g \frac{h^{2}}{2} \\
\partial_{t} \mathbb{P} & +\boldsymbol{u} \cdot \nabla \mathbb{P}+(\nabla \boldsymbol{u}) \mathbb{P}+\mathbb{P}(\nabla \boldsymbol{u})^{\mathrm{T}} & =\mathbb{D}
\end{array}\right.
$$

Here $\boldsymbol{u}$ denotes the depth averaged horizontal velocity, the stress tensor $\mathbb{P}$ quantifies the distortion of the velocity field depending on the depth and $C_{f}$ is a Chézy coefficient. If these equations 
are written in the 2D Cartesian frame $(x, y)$ then the gravity is in the $z$-direction. Therefore, the bottom topography is defined by $z=\mathrm{b}(x, y)$. The tensor $\mathbb{D}$ can be quantified from energy conservation law [7] that the system (2.2) admits in addition to conservation laws for $h$ and $h \boldsymbol{u}$. The energy conservation law is written as:

$$
\partial_{t} E+\nabla \cdot((E+p) \boldsymbol{u}+h \mathbb{P} \boldsymbol{u})=-g h \nabla \mathbf{b} \cdot \boldsymbol{u}-C_{f}|\boldsymbol{u}|^{3}+\frac{h \operatorname{Tr}(\mathbb{D})}{2}
$$

where

$$
E=h e+h e^{\prime}, \quad e=\frac{\boldsymbol{u} \cdot \boldsymbol{u}+g h+\operatorname{Tr}(\mathbb{P})}{2}, \quad p=\frac{g h^{2}}{2} \quad \text { and } \quad \mathbb{D}=-\frac{2 \alpha|\boldsymbol{u}|^{3} \mathbb{P}}{h}
$$

and the term $e^{\prime}$ corresponds to the unresolved energy. The dissipation of $\mathbb{P}$ is exactly compensated by the production of the unresolved energy $e^{\prime}$. The term $\alpha$ in $\mathbb{D}$ is specified as

$$
\alpha=\max \left(0, C_{r} \frac{\operatorname{Tr}(\mathbb{P})-\phi h^{2}}{\operatorname{Tr}(\mathbb{P})^{2}}\right)
$$

where $\phi$ and $C_{r}$ are the constants of the model [13] which denote the enstrophy of small vortices near the bottom and the dissipation coefficient of roller formation, respectively. For a smooth solution, the system nondissipative system admits the conservation of the enstrophy $(\Psi)$

$$
\partial_{t}(h \Psi)+\nabla \cdot(h \Psi \boldsymbol{u})=0
$$

where enstrophy is defined as $\Psi=\frac{\operatorname{det}(\mathbb{P})}{h^{2}}$. This quantity plays the role of mathematical entropy.

The numerical solution of the system (2.1) will be achieved in two main steps associated to the non-dissipative and the purely dissipative parts. The purely dissipative part can be written as:

$$
\begin{cases}\partial_{t} h & =0 \\ \partial_{t}(h \boldsymbol{u}) & =-g h \nabla \mathrm{b} \cdot \boldsymbol{u}-C_{f}|\boldsymbol{u}|^{2} \boldsymbol{u} \\ \partial_{t} \mathbb{P} & =-\frac{2 \alpha}{h}|\boldsymbol{u}|^{3} \mathbb{P}\end{cases}
$$

and can be solved by following the standard splitting approach [16] i.e. by using the solution of non-dissipative part as the initial condition for above system of ordinary differential equations (ODEs) given by (2.4). The system (2.4) does not pose significant numerical difficulties. Henceforth, we will focus upon the non-dissipative part of the model.

\section{Non-dissipative model}

The non-dissipative model for SSWF is written as:

$$
\left\{\begin{array}{lll}
\partial_{t} h & +\nabla \cdot(h \boldsymbol{u}) & =0 \\
\partial_{t}(h \boldsymbol{u}) & +\nabla \cdot(h \boldsymbol{u} \otimes \boldsymbol{u}+p \mathbf{I}+h \mathbb{P}) & =0 \\
\partial_{t} \mathbb{P} & +\boldsymbol{u} \cdot \nabla \mathbb{P}+(\nabla \boldsymbol{u}) \mathbb{P}+\mathbb{P}(\nabla \boldsymbol{u})^{\mathrm{T}} & =
\end{array}\right.
$$

This nonlinear, non-conservative system of hyperbolic of PDE contains five different waves. For a unit vector $(\boldsymbol{n})$ in any direction, the eigenvalues of the system (3.1) are

$$
\lambda_{0}=\boldsymbol{u} \cdot \boldsymbol{n}, \quad \lambda_{ \pm s}=\boldsymbol{u} \cdot \boldsymbol{n} \pm \sqrt{\mathbb{P}:(\boldsymbol{n} \otimes \boldsymbol{n})} \quad \text { and } \quad \lambda_{ \pm a}=\boldsymbol{u} \cdot \boldsymbol{n} \pm \sqrt{g h+3 \mathbb{P}:(\boldsymbol{n} \otimes \boldsymbol{n})}
$$

The eigenvalues $\lambda_{0}, \lambda_{ \pm s}$ and $\lambda_{ \pm a}$ represent the speeds of material, shear and acoustic waves respectively. The first three eigenvalues $\left(\lambda_{0}, \lambda_{ \pm s}\right)$ are linearly degenerate and the two last $\left(\lambda_{ \pm a}\right)$ 
are genuinely nonlinear. Since the tensor $\mathbb{P}$ is always symmetric and positive definite, there is a strict ordering of the eigenvalues

$$
\lambda_{-a}<\lambda_{-s}<\lambda_{0}<\lambda_{s}<\lambda_{a}
$$

A detailed analysis of characteristic structure of the system (3.1) is presented in [7]. Our goal is to develop a numerical approximation of the non-conservative system (3.1) using an approximated Riemann solver in a Godonov-type method [8]. As the system is non-conservative, the Riemann problem can have multiple weak solutions. These solutions depend on the paths those link the different states associated with discontinuities. In the present context, we will design a favorite path based on two ingredients : splitting and the entropy inequality associated to (2.3). Splitting will be used to separate the shear waves in one sub-system and the material and acoustic waves in the other. For the latter sub-system, the conservation of the total energy is used to derive a complete set of jump conditions. These conditions can be used to determine the intermediate states of the associated Riemann problem.

\subsection{Riemann Problem}

Let us represent the velocity vector $\boldsymbol{u}$ and the symmetric stress tensor $\mathbb{P}$ using two orthogonal unit vectors $\boldsymbol{n}$ and $\boldsymbol{m}$ as

$$
\boldsymbol{u}=\boldsymbol{u}_{n} \boldsymbol{n}+\boldsymbol{u}_{m} \boldsymbol{m}, \quad \mathbb{P}=\mathbb{P}_{n n}(\boldsymbol{n} \otimes \boldsymbol{n})+\mathbb{P}_{n m}(\boldsymbol{n} \otimes \boldsymbol{m}+\boldsymbol{m} \otimes \boldsymbol{n})+\mathbb{P}_{m m}(\boldsymbol{m} \otimes \boldsymbol{m})
$$

Therefore, in any local frame $(\boldsymbol{n}, \boldsymbol{m})$ the system $(3.1)$ can be written as

$$
\left\{\begin{array}{llll}
\partial_{t} h & +\partial_{n}\left(h \boldsymbol{u}_{n}\right) & +\partial_{m}\left(h \boldsymbol{u}_{n}\right) & =0 \\
\partial_{t}\left(h \boldsymbol{u}_{n}\right) & +\partial_{n}\left(h \boldsymbol{u}_{n}^{2}+p+h \mathbb{P}_{n n}\right) & +\partial_{m}\left(h \boldsymbol{u}_{m} \boldsymbol{u}_{n}+h \mathbb{P}_{n m}\right) & =0 \\
\partial_{t}\left(h \boldsymbol{u}_{m}\right) & +\partial_{n}\left(h \boldsymbol{u}_{m} \boldsymbol{u}_{n}+h \mathbb{P}_{n m}\right) & +\partial_{m}\left(h \boldsymbol{u}_{m}^{2}+p+h \mathbb{P}_{n m}\right) & =0 \\
\partial_{t} \mathbb{P}_{n n} & +\boldsymbol{u}_{n} \partial_{n} \mathbb{P}_{n n}+2 \mathbb{P}_{n n} \partial_{n} \boldsymbol{u}_{n}+\boldsymbol{u}_{m} \partial_{m} \mathbb{P}_{n n}+2 \mathbb{P}_{n m} \partial_{m} \boldsymbol{u}_{n} & =0 \\
\partial_{t} \mathbb{P}_{m m} & +\boldsymbol{u}_{n} \partial_{n} \mathbb{P}_{m m}+2 \mathbb{P}_{n m} \partial_{n} \boldsymbol{u}_{m} & +\boldsymbol{u}_{m} \partial_{m} \mathbb{P}_{m m}+2 \mathbb{P}_{m m} \partial_{m} \boldsymbol{u}_{m} & =0 \\
\partial_{t} \mathbb{P}_{n m} & +\partial_{n}\left(\boldsymbol{u}_{m} \mathbb{P}_{n m}\right)+\mathbb{P}_{n n} \partial_{n} \boldsymbol{u}_{m} & +\partial_{m}\left(\boldsymbol{u}_{n} \mathbb{P}_{n m}\right)+\mathbb{P}_{n n} \partial_{m} \boldsymbol{u}_{n} & =0
\end{array}\right.
$$

In any direction $\boldsymbol{n}$, the associated Riemann problem can be written as

$$
\left\{\begin{array}{lll}
\partial_{t} h & +\partial_{n}\left(h \boldsymbol{u}_{n}\right) & =0 \\
\partial_{t}\left(h \boldsymbol{u}_{n}\right) & +\partial_{n}\left(h \boldsymbol{u}_{n}^{2}+p+h \mathbb{P}_{n n}\right) & =0 \\
\partial_{t}\left(h \boldsymbol{u}_{m}\right) & +\partial_{n}\left(h \boldsymbol{u}_{m} \boldsymbol{u}_{n}+h \mathbb{P}_{n m}\right) & =0 \\
\partial_{t} \mathbb{P}_{n n}+\boldsymbol{u}_{n} \partial_{n} \mathbb{P}_{n n}+2 \mathbb{P}_{n n} \partial_{n} \boldsymbol{u}_{n} & =0 \\
\partial_{t} \mathbb{P}_{m m}+\boldsymbol{u}_{n} \partial_{n} \mathbb{P}_{m m}+2 \mathbb{P}_{n m} \partial_{n} \boldsymbol{u}_{m}=0 \\
\partial_{t} \mathbb{P}_{n m}+\partial_{n}\left(\boldsymbol{u}_{m} \mathbb{P}_{n m}\right)+\mathbb{P}_{n n} \partial_{n} \boldsymbol{u}_{m}=0
\end{array}\right.
$$

with the initial condition

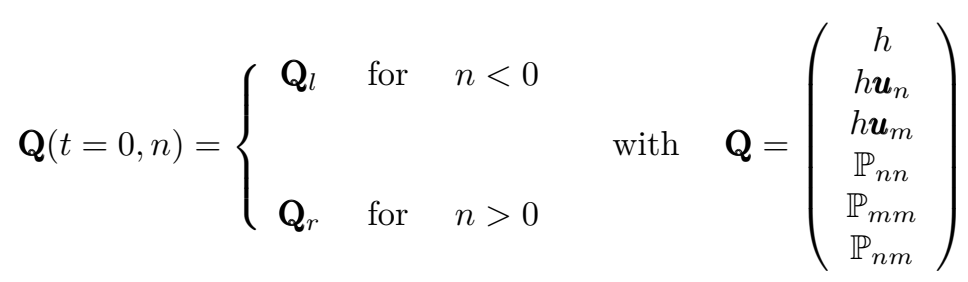

This Riemann problem has a self-similar solution. We assume that there exist a unique "entropic weak" solution composed by simple waves. This system is characterized by five waves with the speeds:

$$
\mathrm{S}_{l} \equiv \lambda_{-a} \quad<\quad \mathrm{s}_{l} \equiv \lambda_{-s} \quad<\quad \mathrm{u}^{s, \star} \equiv \lambda_{0} \quad<\quad \mathrm{s}_{r} \equiv \lambda_{s} \quad<\quad \mathrm{S}_{r} \equiv \lambda_{a}
$$


When there are no rarefaction waves, the solution to the Riemann problem $\left(\mathbf{Q}_{\mathcal{R}}\right)$ is composed by constant states separated by time-space discontinuity lines. Using the similarity variable $\sigma=n / t$, we have

$$
\mathbf{Q}_{\mathcal{R}}(t, n) \equiv \mathbf{Q}_{\mathcal{R}}(\sigma)=\left\{\begin{array}{cll}
\mathbf{Q}_{l} & \text { for } & \sigma<\mathrm{S}_{l} \\
\mathbf{Q}_{l \star} & \text { for } & \mathrm{S}_{l}<\sigma<\mathrm{s}_{l} \\
\mathbf{Q}_{l \star \star} & \text { for } & \mathrm{s}_{l}<\sigma<\mathrm{u}^{s, \star} \\
\mathbf{Q}_{r \star \star} & \text { for } & \mathrm{u}^{s, \star}<\sigma<\mathrm{s}_{r} \\
\mathbf{Q}_{r \star} & \text { for } & \mathrm{s}_{r}<\sigma<\mathrm{S}_{r} \\
\mathbf{Q}_{r} & \text { for } & \mathrm{S}_{r}<\sigma
\end{array}\right.
$$

If an exact or a consistent approximate solution of the Riemann problem is found, a Godunov-type method can be derived [8]. In fact Godunov methods are based on the fluctuations associated to the Riemann problem. These left-going $\boldsymbol{\psi}_{l}$ and right-going $\boldsymbol{\psi}_{r}$ fluctuations are given by

$$
\begin{aligned}
& \psi_{l}\left(\mathbf{Q}_{l}, \mathbf{Q}_{r}\right)=\frac{1}{t} \int_{n<0}\left(\mathbf{Q}_{\mathcal{R}}(t, n)-\mathbf{Q}_{l}\right) d n=\int_{n<0}\left(\mathbf{Q}_{\mathcal{R}}(\sigma)-\mathbf{Q}_{l}\right) d \sigma \\
& \boldsymbol{\psi}_{r}\left(\mathbf{Q}_{l}, \mathbf{Q}_{r}\right)=\frac{1}{t} \int_{n>0}\left(\mathbf{Q}_{r}-\mathbf{Q}_{\mathcal{R}}(t, n)\right) d n=\int_{n>0}\left(\mathbf{Q}_{r}-\mathbf{Q}_{\mathcal{R}}(\sigma)\right) d \sigma
\end{aligned}
$$

The exact computation of the different intermediate states is out of the scope of this paper. In next sub-sections, we focus on the approximation of the fluctuations $\psi$ using a splitting strategy. The approach of preferable "family of paths" is used here, even if an explicit formulation of the path is not provided.

\subsection{Splitting}

The system (3.3) is a case of a non-conservative hyperbolic system whose characteristic matrix can be written as $\mathcal{A}=\mathcal{H} \Lambda \mathcal{H}^{-1}$, where $\Lambda(\mathbf{Q})$ and $\mathcal{H}(\mathbf{Q})$ are matrices of the eigenvalues (diagonal) and the eigenvectors, respectively. We can write the system (3.3) in a quasi-linear form

$$
\frac{\partial \mathbf{Q}}{\partial t}+\mathcal{H} \Lambda \mathcal{H}^{-1} \frac{\partial \mathbf{Q}}{\partial n}=0
$$

We use principle of splitting to decompose $\Lambda$ into the sum of diagonal matrices corresponding to the each sub-system of (3.3). This decomposition aims to separate the eigenvalues in such a way that a Riemann solver for each sub-system can be easily derived. The following decomposition is used to separate the shear waves from acoustic and material waves:

$$
\Lambda=\Lambda^{s}+\Lambda^{a}
$$

where

$$
\Lambda^{s}=\operatorname{diag}\left(\begin{array}{c}
0 \\
-\sqrt{\mathbb{P}:(\boldsymbol{n} \otimes \boldsymbol{n})}, \\
0 \\
0 \\
\sqrt{\mathbb{P}:(\boldsymbol{n} \otimes \boldsymbol{n})} \\
0
\end{array}\right) \quad \text { and } \quad \Lambda^{a}=\operatorname{diag}\left(\begin{array}{c}
\boldsymbol{u} \cdot \boldsymbol{n}-\sqrt{g h+3 \mathbb{P}:(\boldsymbol{n} \otimes \boldsymbol{n})} \\
\boldsymbol{u} \cdot \boldsymbol{n} \\
\boldsymbol{u} \cdot \boldsymbol{n} \\
\boldsymbol{u} \cdot \boldsymbol{n} \\
\boldsymbol{u} \cdot \boldsymbol{n} \\
\boldsymbol{u} \cdot \boldsymbol{n}+\sqrt{g h+3 \mathbb{P}:(\boldsymbol{n} \otimes \boldsymbol{n})}
\end{array}\right)
$$


Then the shear sub-system (associated to $\Lambda^{s}$ ) and acoustic sub-system (associated to $\Lambda^{a}$ ) are

$$
\frac{\partial \mathbf{Q}}{\partial t}+\mathcal{H} \Lambda^{s} \mathcal{H}^{-1} \frac{\partial \mathbf{Q}}{\partial n}=0 \quad \text { and } \quad \frac{\partial \mathbf{Q}}{\partial t}+\mathcal{H} \Lambda^{a} \mathcal{H}^{-1} \frac{\partial \mathbf{Q}}{\partial n}=0
$$

These sub-systems are hyperbolic on the account of their formulation. The shear sub-system can be written as

$$
\begin{cases}\partial_{t} h & =0 \\ \partial_{t}\left(h \boldsymbol{u}_{n}\right) & =0 \\ \partial_{t}\left(h \boldsymbol{u}_{m}\right)+\partial_{n}\left(h \mathbb{P}_{n m}\right) & =0 \\ \partial_{t} \mathbb{P}_{n n} & =0 \\ \partial_{t} \mathbb{P}_{m m}+2 \mathbb{P}_{n m} \partial_{n} \boldsymbol{u}_{m} & =0 \\ \partial_{t} \mathbb{P}_{n m}+\mathbb{P}_{n n} \partial_{n} \boldsymbol{u}_{m} & =0\end{cases}
$$

and the acoustic sub-system can be written as

$$
\left\{\begin{array}{lll}
\partial_{t} h & +\partial_{n}\left(h \boldsymbol{u}_{n}\right) & =0 \\
\partial_{t}\left(h \boldsymbol{u}_{n}\right) & +\partial_{n}\left(h \boldsymbol{u}_{n}^{2}+p+h \mathbb{P}_{n n}\right) & =0 \\
\partial_{t}\left(h \boldsymbol{u}_{m}\right) & +\partial_{n}\left(h \boldsymbol{u}_{m} \boldsymbol{u}_{n}\right) & =0 \\
\partial_{t} \mathbb{P}_{n n}+\boldsymbol{u}_{n} \partial_{n} \mathbb{P}_{n n}+2 \mathbb{P}_{n n} \partial_{n} \boldsymbol{u}_{n} & =0 \\
\partial_{t} \mathbb{P}_{m m}+\boldsymbol{u}_{n} \partial_{n} \mathbb{P}_{m m} & =0 \\
\partial_{t} \mathbb{P}_{n m}+\partial_{n}\left(\boldsymbol{u}_{m} \mathbb{P}_{n m}\right) & =0
\end{array}\right.
$$

Let us denote by $\psi^{s}$ and $\psi^{a}$ the partial fluctuations associated to the shear (3.9) and acoustic sub-system (3.10), respectively. Therefore, the fluctuation associated to the system (3.3) can be approximated by

$$
\psi \simeq \psi^{s}+\psi^{a}
$$

The construction of each partial fluctuation is shown in the next sub-sections.

\subsection{Exact Riemann solver for the shear sub-system}

Here, we rewrite the eigenvalues of the shear sub-system as:

$$
\lambda_{-}^{s}=-\sqrt{\mathbb{P}_{n n}}<\lambda_{0}^{s}=0 \quad<\lambda_{+}^{s}=+\sqrt{\mathbb{P}_{n n}}
$$

All of these eigenvalues are linearly degenerated. Therefore, we can compute the following set of twelve Riemann invariants

$$
\left\{\begin{aligned}
\lambda_{-}^{s}: & I_{1}^{-}=h, \quad I_{2}^{-}=\boldsymbol{u}_{m}, \quad I_{3}^{-}=\mathbb{P}_{n m}, \quad I_{4}^{-}=\boldsymbol{u}_{m}-\frac{\mathbb{P}_{n m}}{\lambda_{-}}, \quad I_{5}^{-}=\mathbb{P}_{n m}^{2}-\lambda_{-}^{2} \mathbb{P}_{n m} \\
\lambda_{0}^{s}: & I_{1}^{0}=\boldsymbol{u}_{m}, \quad I_{2}^{0}=h \mathbb{P}_{n n} \\
\lambda_{+}^{s}: & I_{1}^{+}=h, \quad I_{2}^{+}=\boldsymbol{u}_{m}, \quad I_{3}^{+}=\mathbb{P}_{n m}, \quad I_{4}^{+}=\boldsymbol{u}_{m}-\frac{\mathbb{P}_{n m}}{\lambda_{+}}, \quad I_{5}^{+}=\mathbb{P}_{n m}^{2}-\lambda_{+}^{2} \mathbb{P}_{n m}
\end{aligned}\right.
$$

Using these Riemann invariants, it is possible to define the twelve variables associated to the two intermediate states of the Riemann problem. For a given left and right states, the solution to the Riemann problem is defined by the following four states :

$$
\mathbf{Q}_{\mathcal{R}}^{s}(t, n)=\left\{\begin{array}{ll}
\mathbf{Q}_{l} & \text { for } \quad \sigma<\mathrm{s}_{l} \\
\mathbf{Q}_{l}^{s, \star} & \text { for } \quad \mathrm{s}_{l}<\sigma<\mathrm{u}^{s, \star}=0 \\
\mathbf{Q}_{r}^{s, \star} & \text { for } \mathrm{u}^{s, \star}=0<\sigma<\mathrm{s}_{r} \\
\mathbf{Q}_{r} & \text { for } \quad \mathrm{s}_{r}<\sigma
\end{array} \quad \text { with } \quad \sigma=\frac{n}{t}\right.
$$


where

$$
\mathbf{Q}_{l}=\left(\begin{array}{c}
h_{l} \\
h_{l} \boldsymbol{u}_{n, l} \\
h_{l} \boldsymbol{u}_{m, l} \\
\mathbb{P}_{n n, l} \\
\mathbb{P}_{m m, l} \\
\mathbb{P}_{n m, l}
\end{array}\right), \quad \mathbf{Q}_{l}^{s, \star}=\left(\begin{array}{c}
h_{l} \\
h_{l} \boldsymbol{u}_{n, l} \\
h_{l} \boldsymbol{u}_{m}^{s, \star} \\
\mathbb{P}_{n n, l} \\
\mathbb{P}_{m m, l}^{s, \star} \\
\beta^{s, \star} / h_{l}
\end{array}\right), \quad \mathbf{Q}_{r}^{s, \star}=\left(\begin{array}{c}
h_{r} \\
h_{r} \boldsymbol{u}_{n, r} \\
h_{r} \boldsymbol{u}_{m, \star}^{s, \star} \\
\mathbb{P}_{n n, r} \\
\mathbb{P}_{m, \star}^{s, \star}, r, r \\
\beta^{s, \star} / h_{r}
\end{array}\right), \quad \mathbf{Q}_{r}=\left(\begin{array}{c}
h_{r} \\
h_{r} \boldsymbol{u}_{n, r} \\
h_{r} \boldsymbol{u}_{m, r} \\
\mathbb{P}_{n n, r} \\
\mathbb{P}_{m m, r} \\
\mathbb{P}_{n m, r}
\end{array}\right)
$$

where $\beta^{s, \star}=\left(h \mathbb{P}_{n m}\right)_{l}^{s, \star}=\left(h \mathbb{P}_{n m}\right)_{r}^{s, \star}$ and the states are separated by the waves with the speeds

$$
\mathrm{s}_{l}=-\sqrt{\mathbb{P}_{n n, l}}<\mathrm{s}_{0}=0<\mathrm{s}_{r}=\sqrt{\mathbb{P}_{n n, r}}
$$

For the left and right waves associated to $\lambda=\mathrm{s}_{l}<0$ and $\lambda=\mathrm{s}_{r}>0$, the intermediate states are completely defined by the following additional relations :

$$
\begin{aligned}
h_{l} \mathrm{~s}_{l} \boldsymbol{u}_{m}^{s, \star}-\beta^{s, \star} & =h_{l} \mathrm{~s}_{l} \boldsymbol{u}_{m, l}-h_{l} \mathbb{P}_{n m, l} \\
\left(\beta^{s, \star}\right)^{2}-\mathrm{s}_{l}^{2} h_{l}^{2} \mathbb{P}_{m m, l}^{s,} & =h_{l}^{2}\left(\mathbb{P}_{n m, l}^{2}-\mathrm{s}_{l}^{2} \mathbb{P}_{m m, l}\right) \\
h_{r} \mathrm{~s}_{r} \boldsymbol{u}_{m}^{s, \star}-\beta^{s, \star} & =h_{r} \mathrm{~s}_{r} \boldsymbol{u}_{m, r}-h_{r} \mathbb{P}_{n m, r} \\
\left(\beta^{s, \star}\right)^{2}-\mathrm{s}_{r}^{2} h_{r}^{2} \mathbb{P}_{m m, r}^{s, \star} & =h_{r}^{2}\left(\mathbb{P}_{n m, r}^{2}-\mathrm{s}_{r}^{2} \mathbb{P}_{m m, r}\right)
\end{aligned}
$$

After a straight-forward algebra, we can express the stared variables of the intermediate state as functions of the left and right states of the Riemann problem.

$$
\left\{\begin{aligned}
\boldsymbol{u}_{m}^{s, \star} & =\frac{\mathrm{s}_{r} h_{r} \boldsymbol{u}_{m, r}-\mathrm{s}_{l} h_{l} \boldsymbol{u}_{m, l}+\left(h_{r} \mathbb{P}_{n m, r}-h_{l} \mathbb{P}_{n m, l}\right)}{h_{r} \mathrm{~s}_{r}-h_{l} \mathrm{~s}_{l}} \\
\beta^{s, \star} & =h_{l} h_{r} \frac{\mathrm{s}_{r}\left(\mathbb{P}_{n m, l}-\mathrm{s}_{l} \boldsymbol{u}_{m, l}\right)-\mathrm{s}_{l}\left(\mathbb{P}_{n m, r}+\mathrm{s}_{r} \boldsymbol{u}_{m, r}\right)}{h_{r} \mathrm{~s}_{r}-h_{l} \mathrm{~s}_{l}} \\
\mathbb{P}_{m m, l}^{s, \star} & =\frac{\left(\beta^{s, \star}\right)^{2}-h_{l}^{2}\left(\mathbb{P}_{n m, l}^{2}-\mathrm{s}_{l}^{2} \mathbb{P}_{m m, l}\right)}{\mathrm{s}_{l}^{2} h_{l}^{2}} \\
\mathbb{P}_{m m, r}^{s, \star} & =\frac{\left(\beta^{s, \star}\right)^{2}-h_{r}^{2}\left(\mathbb{P}_{n m, r}^{2}-\mathrm{s}_{r}^{2} \mathbb{P}_{m m, r}\right)}{\mathrm{s}_{r}^{2} h_{r}^{2}}
\end{aligned}\right.
$$

Therefore, the fluctuations associated with shear sub-system can be written as:

$$
\begin{aligned}
& \boldsymbol{\psi}_{l}^{s}=-\mathrm{s}_{l}^{-}\left(\mathbf{Q}_{l}^{s, \star}-\mathbf{Q}_{l}\right)-\mathrm{s}_{0}^{-}\left(\mathbf{Q}_{r}^{s, \star}-\mathbf{Q}_{l}^{s, \star}\right)-\mathrm{s}_{r}^{-}\left(\mathbf{Q}_{r}-\mathbf{Q}_{r}^{s, \star}\right) \\
& \boldsymbol{\psi}_{r}^{s}=\mathrm{s}_{l}^{+}\left(\mathbf{Q}_{l}^{s, \star}-\mathbf{Q}_{l}\right)+\mathrm{s}_{0}^{+}\left(\mathbf{Q}_{r}^{s, \star}-\mathbf{Q}_{l}^{s, \star}\right)+\mathrm{s}_{r}^{+}\left(\mathbf{Q}_{r}-\mathbf{Q}_{r}^{s, \star}\right)
\end{aligned}
$$

where $\mathrm{s}^{-}=\min (0, \mathrm{~s})$ and $\mathrm{s}^{+}=\max (0, \mathrm{~s})$.

\subsection{HLL-like Riemann solver for the acoustic sub-system (3.10).}

The eigenvalues for the acoustic sub-system are

$$
\lambda_{-}^{a}=\boldsymbol{u}_{n}-\sqrt{g h+3 \mathbb{P}_{n n}}<\lambda_{0}^{a}=\boldsymbol{u}_{n} \quad \lambda_{+}^{a}=\boldsymbol{u}_{n}+\sqrt{g h+\mathbb{P}_{n n}}
$$

The eigenvalue $\lambda_{0}^{a}$ is linearly degenerate for two Riemann invariants, normal velocity $\boldsymbol{u}_{n}$ and the total pressure $\pi=g \frac{h^{2}}{2}+h \mathbb{P}_{n n}$. On the other hand, the eigenvalues $\lambda_{ \pm}^{a}$ are genuinely nonlinear and can develop either shocks (discontinuities) or rarefactions (smooth waves). The derivation of an exact Riemann solver is tricky when there are shocks and rarefactions and it will not be considered here. An approximate Riemann solver is our target for the acoustic sub-system. Let 
us consider an consistent approximation in which the nonlinear wave speeds are denoted by $\mathrm{S}_{l}$ and $S_{r}$. In this context, the approximate Riemann problem is formulated by the following four states:

$$
\mathbf{Q}_{\mathcal{R}}^{a}(t, n)=\left\{\begin{array}{cll}
\mathbf{Q}_{l} & \text { for } & \sigma<\mathrm{S}_{l} \\
\mathbf{Q}_{l}^{a, \star} & \text { for } & \mathrm{S}_{l}<\sigma<\mathrm{u}^{a, \star} \\
\mathbf{Q}_{r}^{a, \star} & \text { for } & \mathrm{u}^{a, \star}<\sigma<\mathrm{S}_{r} \\
\mathbf{Q}_{r} & \text { for } & \mathrm{S}_{r}<\sigma
\end{array} \quad \text { with } \quad \sigma=\frac{n}{t}\right.
$$

where

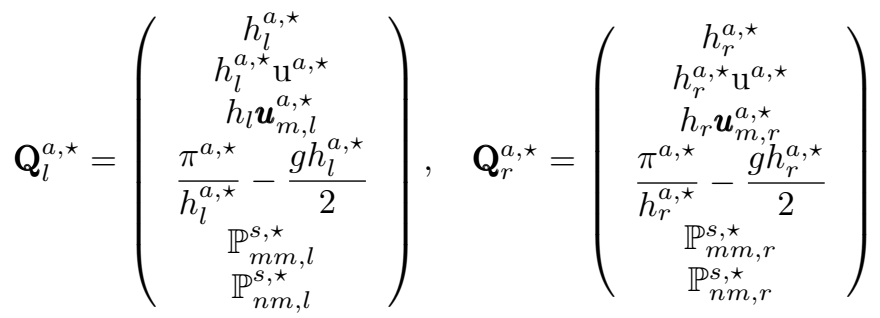

The intermediates states are formulated by ten unknowns:

$$
h_{l}^{a, \star}, \quad \mathrm{u}^{a, \star}, \quad \boldsymbol{u}_{m, l}^{a, \star}, \quad \pi^{a, \star}, \quad \mathbb{P}_{m m, l}^{s, \star}, \quad \mathbb{P}_{n m, l}^{s, \star}, \quad h_{r}^{a, \star}, \quad \boldsymbol{u}_{m, r}^{a, \star}, \quad \mathbb{P}_{m m, r}^{s, \star}, \quad \mathbb{P}_{n m, r}^{s, \star}
$$

In order to express these unknowns in terms of the initial condition of the Riemann solver, we need to find ten independents relations. Since, the evolution of the depth $(h)$, momentum $(h \boldsymbol{u})$ and $\mathbb{P}_{n m}$ are governed by conservation equations, Rankine-Hugoniot conditions for left and right acoustic waves give

$$
\left\{\begin{aligned}
\mathrm{S}_{l}\left(h_{l}^{a, \star}-h_{l}\right) & =h_{l}^{a, \star} \mathrm{u}^{a, \star}-\left(h \boldsymbol{u}_{n}\right)_{l} \\
\mathrm{~S}_{l}\left(h_{l}^{a, \star} \mathrm{u}^{a, \star}-\left(h \boldsymbol{u}_{n}\right)_{l}\right) & =\left(h_{l}^{a, \star}\left(\mathrm{u}^{a, \star}\right)^{2}+\pi^{a, \star}\right)-\left(h \boldsymbol{u}_{n}^{2}+\pi\right)_{l} \\
\mathrm{~S}_{l}\left(h_{l}^{a, \star} \boldsymbol{u}_{m, l}^{a, \star}-\left(h \boldsymbol{u}_{m}\right)_{l}\right) & =\left(h_{l}^{a, \star} \mathrm{u}^{a, \star} \boldsymbol{u}_{m, l}^{a, \star}-h_{l} \boldsymbol{u}_{n, l} \boldsymbol{u}_{m, l}\right) \\
\mathrm{S}_{l}\left(\mathbb{P}_{n m, l}^{a, \star}-\mathbb{P}_{n m, l}\right) & =\left(\boldsymbol{u}_{n, l}^{a, \star} \mathbb{P}_{n m, l}^{a, \star}-\boldsymbol{u}_{n, l} \mathbb{P}_{n m, l}\right)
\end{aligned}\right.
$$

and

$$
\left\{\begin{aligned}
\mathrm{S}_{r}\left(h_{r}^{a, \star}-h_{r}\right) & =h_{r}^{a, \star} \mathrm{u}^{a, \star}-\left(h \boldsymbol{u}_{n}\right)_{r} \\
\mathrm{~S}_{r}\left(h_{r}^{a, \star} \mathrm{u}^{a, \star}-\left(h \boldsymbol{u}_{n}\right)_{r}\right) & =\left(h_{r}^{a, \star}\left(\mathrm{u}^{a, \star}\right)^{2}+\pi^{a, \star}\right)-\left(h \boldsymbol{u}_{n}^{2}+\pi\right)_{r} \\
\mathrm{~S}_{r}\left(h_{r}^{a, \star} \boldsymbol{u}_{m, r}^{a, \star}-\left(h \boldsymbol{u}_{m}\right)_{r}\right) & =\left(h_{r}^{a, \star} \mathrm{u}^{a, \star} \boldsymbol{u}_{m, r}^{a, \star}-h_{r} \boldsymbol{u}_{n, r} \boldsymbol{u}_{m, r}\right) \\
\mathrm{S}_{r}\left(\mathbb{P}_{n m, r}^{a, \star}-\mathbb{P}_{n m, r}\right) & =\left(\boldsymbol{u}_{n, r}^{a, \star} \mathbb{P}_{n m, r}^{a, \star}-\boldsymbol{u}_{n, r} \mathbb{P}_{n m, r}\right)
\end{aligned}\right.
$$

From these relations, we obtain

$$
\begin{gathered}
\mathrm{u}^{a, \star}=\frac{\mathrm{m}_{r} \boldsymbol{u}_{n, r}-\mathrm{m}_{l} \boldsymbol{u}_{n, l}+\pi_{r}-\pi_{l}}{\mathrm{~m}_{r}-\mathrm{m}_{l}} \\
\pi^{a, \star}=\frac{\mathrm{m}_{r} \pi_{l}-\mathrm{m}_{l} \pi_{r}-\mathrm{m}_{r} \mathrm{~m}_{l}\left(\boldsymbol{u}_{n, r}-\boldsymbol{u}_{n, l}\right)}{\mathrm{m}_{r}-\mathrm{m}_{l}} \\
\left\{\begin{array} { r l } 
{ h _ { l } ^ { a , \star } } & { = \frac { \mathrm { m } _ { l } } { \mathrm { u } ^ { a , \star } - \mathrm { S } _ { l } } } \\
{ \boldsymbol { u } _ { m , l } ^ { a , \star } } & { = \boldsymbol { u } _ { m , l } } \\
{ \mathbb { P } _ { n m , l } ^ { a , \star } } & { = \frac { h _ { l } ^ { a , \star } } { h _ { l } } \mathbb { P } _ { n m , l } }
\end{array} \quad \text { and } \left\{\begin{array}{rl}
h_{r}^{a, \star} & =\frac{\mathrm{m}_{r}}{\boldsymbol{u}_{m, r}^{a, \star}}=\mathrm{u}_{m, r} \\
\mathbb{P}_{n m, r}^{a, \star} & =\frac{h_{r}^{a, \star}}{h_{r}} \mathbb{P}_{n m, r}
\end{array}\right.\right.
\end{gathered}
$$


where $\mathrm{m}_{l}=h_{l}\left(\boldsymbol{u}_{n, l}-\mathrm{S}_{l}\right)$ and $\mathrm{m}_{r}=h_{r}\left(\boldsymbol{u}_{n, r}-\mathrm{S}_{r}\right)$. The jump conditions across a shock with the speed $S$ can be written as

$$
S[h]=\left[h \boldsymbol{u}_{n}\right], \quad S\left[h \boldsymbol{u}_{n}\right]=\left[h \boldsymbol{u}_{n}^{2}+\pi\right]
$$

This imply $[\mathrm{m}]=0$ and $\mathrm{m}\left[\boldsymbol{u}_{n}\right]=-[\pi]$, where $\mathrm{m}=h\left(\boldsymbol{u}_{n}-S\right)$. As $\left[\boldsymbol{u}_{n}\right]=\left[\frac{\mathrm{m}}{h}\right]$ and with $\vartheta=\frac{1}{h}$, we can write

$$
-\mathrm{m}^{2}[\vartheta]=[\pi] .
$$

This means that $\pi$ and $h$ are both either increasing or decreasing across the shocks. On the other hand

$$
\begin{gathered}
{\left[\frac{\mathbb{P}_{n n}}{h^{2}}\right]=\left(\overline{\vartheta^{3}}-3 \frac{\bar{\pi} \widetilde{\vartheta^{2}}}{\mathrm{~m}^{2}}+\frac{g}{2 \mathrm{~m}^{2}}\right)[\pi] \quad \text { and } \quad\left[\frac{\mathbb{P}_{n n}}{h}\right]=\left(\overline{\vartheta^{2}}-2 \frac{\bar{\pi} \bar{\vartheta}}{\mathrm{m}^{2}}\right)[\pi]} \\
{\left[\frac{\mathbb{P}_{n n}}{h^{2}}\right]=\left[\pi \vartheta^{3}\right]-\frac{g}{2}[\vartheta]=\overline{\vartheta^{3}}[\pi]+\bar{\pi}\left[\vartheta^{3}\right]+\frac{g}{2 \mathrm{~m}^{2}}[\pi]=\overline{\vartheta^{3}}[\pi]+3 \bar{\pi} \widetilde{\vartheta^{2}}[\vartheta]+\frac{g}{2 \mathrm{~m}^{2}}[\pi]} \\
=\left(\overline{\vartheta^{3}}-3 \frac{\bar{\pi} \vartheta^{2}}{\mathrm{~m}^{2}}+\frac{g}{2 \mathrm{~m}^{2}}\right)[\pi] \\
{\left[\frac{\mathbb{P}_{n n}}{h}\right]=\left[\pi \vartheta^{2}\right]=\overline{\vartheta^{2}}[\pi]+\bar{\pi}\left[\vartheta^{2}\right]=\overline{\vartheta^{2}}[\pi]+2 \bar{\pi} \bar{\vartheta}[\vartheta]} \\
=\left(\overline{\vartheta^{2}}-2 \frac{\bar{\pi} \bar{\vartheta}}{\mathrm{m}^{2}}\right)[\pi]
\end{gathered}
$$

where, for the upstream(u) and downstream(d) states we have

$$
\bar{X}=\frac{X_{u}-X_{d}}{2}, \quad \widetilde{\vartheta^{2}}=\frac{\vartheta_{u}^{2}+\vartheta_{u} \vartheta_{d}+\vartheta_{d}^{2}}{3}
$$

Therefore, the following inequalities are equivalent

$$
\overline{\vartheta^{3}}+\frac{g}{2 \mathrm{~m}^{2}}-3 \frac{\widetilde{\pi \vartheta^{2}}}{\mathrm{~m}^{2}} \geq 0 \Longleftrightarrow\left[\frac{\mathbb{P}_{n n}}{h^{2}}\right][\pi] \geq 0
$$

Now, we need two additional independents relations for remaining two variables. The problem we have to face is that the equations for $\mathbb{P}_{n n}$ and $\mathbb{P}_{m m}$ are in non-conservative form. In these cases, we have to define the preferable "family of paths". These paths, in the phase space, will connect the two states on either side of the shock. In the local frame the enstrophy $\Psi$ can be written as :

$$
\Psi=\frac{\mathbb{P}_{n n}}{h^{2}} \mathbb{P}_{m m}-\left(\frac{\mathbb{P}_{n m}}{h}\right)^{2}
$$

For the acoustic sub-system, there is no jump of $\frac{\mathbb{P}_{n m}}{h}$ across shocks. Therefore the jump of the enstrophy is related to the behavior of $h, \mathbb{P}_{n n}$ and $\mathbb{P}_{m m}$. We need an additional ingredient to find a approximate relation across shocks. For that we write

$$
[\Psi]=\overline{\left(\mathbb{P}_{m m}\right)}\left[\frac{\mathbb{P}_{n n}}{h^{2}}\right]+\overline{\left(\frac{\mathbb{P}_{n n}}{h^{2}}\right)}\left[\mathbb{P}_{m m}\right]
$$

In order to enforce the entropy inequality, at least when $\frac{\mathbb{P}_{n n}}{h^{2}}$ increase across shocks, we consider $\mathbb{P}_{m m}$ is constant across shocks. This consideration gives two relations

$$
\mathbb{P}_{m m, l}^{a, \star}=\mathbb{P}_{m m, l} \quad \text { and } \quad \mathbb{P}_{m m, r}^{a, \star}=\mathbb{P}_{m m, r}
$$


With these additional relations, the intermediate states of the approximate Riemann solver for acoustic sub-system is now completely defined in terms of the initial condition. Therefore, the associated fluctuations can be written as

$$
\begin{aligned}
\boldsymbol{\psi}_{l}^{a} & =-\mathrm{S}_{l}^{-}\left(\mathbf{Q}_{l}^{a, \star}-\mathbf{Q}_{l}\right)-\left(\mathrm{u}^{a, \star}\right)^{-}\left(\mathbf{Q}_{r}^{a, \star}-\mathbf{Q}_{l}^{a, \star}\right)-\mathrm{S}_{r}^{-}\left(\mathbf{Q}_{r}-\mathbf{Q}_{r}^{a, \star}\right) \\
\boldsymbol{\psi}_{r}^{a} & =\mathrm{S}_{l}^{+}\left(\mathbf{Q}_{l}^{a, \star}-\mathbf{Q}_{l}\right)+\left(\mathrm{u}^{a, \star}\right)^{+}\left(\mathbf{Q}_{r}^{a, \star}-\mathbf{Q}_{l}^{s, \star}\right)+\mathrm{S}_{r}^{+}\left(\mathbf{Q}_{r}-\mathbf{Q}_{r}^{a, \star}\right)
\end{aligned}
$$

where $\mathrm{S}^{-}=\min (0, \mathrm{~S})$ and $\mathrm{S}^{+}=\max (0, \mathrm{~S})$.

\subsection{Fluctuation Splitting solver}

In this sub-section, we propose an extended Riemann solver for the non-conservative system (3.1). According to the analysis performed in the previous sub-sections, it is advisable to formulate this solver in terms of fluctuations $\boldsymbol{\Psi}$ in the Cartesian frame. Let us consider the Riemann problem in the direction $\boldsymbol{n}$ with initial conditions defined by the states $\mathbf{U}_{l}$ and $\mathbf{U}_{r}$ in the Cartesian frame. As the transformation from the local to the Cartesian frame is linear, we have

$$
\begin{aligned}
\boldsymbol{\Psi}_{l}\left(\boldsymbol{n}, \mathbf{U}_{l}, \mathbf{U}_{r}\right)= & -\mathrm{s}_{l}^{-}\left(\mathbf{U}_{l}^{s, \star}-\mathbf{U}_{l}\right)-\mathrm{s}_{r}^{-}\left(\mathbf{U}_{r}-\mathbf{U}_{r}^{s, \star}\right) \\
& -\mathrm{S}_{l}^{-}\left(\mathbf{U}_{l}^{a, \star}-\mathbf{U}_{l}\right)-\left(\mathrm{u}^{a, \star}\right)^{-}\left(\mathbf{U}_{r}^{a, \star}-\mathbf{U}_{l}^{s, \star}\right)-\mathrm{S}_{r}^{-}\left(\mathbf{U}_{r}-\mathbf{U}_{r}^{a, \star}\right)
\end{aligned}
$$

and

$$
\begin{aligned}
\boldsymbol{\Psi}_{r}\left(\boldsymbol{n}, \mathbf{U}_{r}, \mathbf{U}_{l}\right)= & \mathrm{s}_{l}^{+}\left(\mathbf{U}_{l}^{s, \star}-\mathbf{U}_{l}\right)+\mathrm{s}_{r}^{+}\left(\mathbf{U}_{r}-\mathbf{U}_{r}^{s, \star}\right) \\
& +\mathrm{S}_{l}^{+}\left(\mathbf{U}_{l}^{a, \star}-\mathbf{U}_{l}\right)+\left(\mathrm{u}^{a, \star}\right)^{+}\left(\mathbf{U}_{r}^{a, \star}-\mathbf{U}_{l}^{s, \star}\right)+\mathrm{S}_{r}^{+}\left(\mathbf{U}_{r}-\mathbf{U}_{r}^{a, \star}\right)
\end{aligned}
$$

This fluctuation formulation is very helpful for non-conservative system where flux formulation is no more valid. For conservative equations with a flux $\mathbf{f}(\mathbf{U})$, the consistency of the Riemann solver with the integral form gives

$$
\boldsymbol{\Psi}_{r}-\mathbf{f}_{r}=\Psi_{l}-\mathbf{f}_{l} \equiv \phi_{l r}\left(\boldsymbol{n}, \mathbf{U}_{l}, \mathbf{U}_{r}\right)
$$

where $\phi_{l r}$ is the classical numerical flux at the interface.

Let us also emphasize that, even if we use almost similar ingredients as in [7], the proposed strategy is completely different. In [7] the link between the decomposition by direction and the ingredients of the solver seem unwavering. This comes principally from the fact that updated variables in the Cartesian frame are nonlinear functions of the variables in the local frames. For example, the local variables for $\mathbb{P}$ are $\widetilde{\mathbb{P}}_{n n}=h \mathbb{P}_{n n}, \widetilde{\mathbb{P}}_{n m}=\mathbb{P}_{n m}$ and $\widetilde{\mathbb{P}}_{n n}=h \mathbb{P}_{n n}$. Therefore, in the Cartesian frame, we have

$$
\mathbb{P}=\frac{\widetilde{\mathbb{P}}_{n n}}{h}(\boldsymbol{n} \otimes \boldsymbol{n})+\widetilde{\mathbb{P}}_{n m}(\boldsymbol{n} \otimes \boldsymbol{m}+\boldsymbol{m} \otimes \boldsymbol{n})+\frac{\widetilde{\mathbb{P}}_{m m}}{h}(\boldsymbol{m} \otimes \boldsymbol{m})
$$

We can observe that with this set of local variables used in [7], it is almost impossible to obtain a linear transformation from a local to Cartesian frame. Therefore the use of unstructured grids will be tricky to adapt with the proposed strategy in [7]. In the approach we propose here, the local Riemann solver is obtained by splitting and is directly formulated in a fixed frame (Cartesian for instance). Therefore one can easily make use of unstructured grids.

\section{$4 \quad$ Numerical Strategy}

\subsection{One dimensional (1D) case}

In $1 \mathrm{D}$ context, we can use $n \equiv x$ and assume that there is no variation in the $m$ direction. The cell volume $\left[x_{i-1 / 2}, x_{i+1 / 2}\right]$ is divided into two sub-cells $\left[x_{i-1 / 2}, x_{i}\right]$ and $\left[x_{i}, x_{i+1 / 2}\right]$, where 
$x_{i \pm 1 / 2}=x_{i} \pm \delta x_{i}$. The sub-cell volumes, for a regular grid, are $a_{i j}=\frac{\delta x}{2}$ for $j=i+1$ and $j=i-1$. At the a given time $t^{n}$ the solution is piece-wise constant and takes the value $\mathbf{U}_{i}^{n}$ in each cell. We assume that the waves associated with the Riemann problem at any interface do not interact with the others. According to the Godunov-type method, the solution is updated at the next time step $t^{n+1}$ as follows

$$
\delta x \mathbf{U}_{i}^{n+1}=\delta x \mathbf{U}_{i}^{n}+\int_{\boldsymbol{x}_{i-1 / 2}}^{\boldsymbol{x}_{i}}\left(\mathbf{U}_{i-1 / 2}\left(t^{n+1}, n\right)-\mathbf{U}_{i}^{n}\right) d x-\int_{\boldsymbol{x}_{i}}^{\boldsymbol{x}_{i+1 / 2}}\left(\mathbf{U}_{i}^{n}-\mathbf{U}_{i+1 / 2}\left(t^{n+1}, n\right)\right) d x
$$

Integrals in above equation are proportional to the fluctuations related to the local Riemann problem. Therefore, the first order explicit scheme is given by

$$
\frac{\mathbf{U}_{i}^{n+1}-\mathbf{U}_{i}^{n}}{\delta t}+\frac{\mathbf{\Psi}_{i+1 / 2}\left(\mathbf{U}_{i}^{n}, \mathbf{U}_{i+1}^{n}\right)-\mathbf{\Psi}_{i-1 / 2}\left(\mathbf{U}_{i}^{n}, \mathbf{U}_{i-1}^{n}\right)}{\delta x}=0
$$

where

and

$$
\mathbf{\Psi}_{i+1 / 2}\left(\mathbf{U}_{i}^{n}, \mathbf{U}_{i+1}^{n}\right) \simeq \frac{1}{\delta t} \int_{\boldsymbol{x}_{i}}^{\boldsymbol{x}_{i+1 / 2}}\left(\mathbf{U}_{i}^{n}-\mathbf{U}_{i+1 / 2}\left(t^{n+1}, x\right)\right) d x
$$

$$
\Psi_{i-1 / 2}\left(\mathbf{U}_{i}^{n}, \mathbf{U}_{i-1}^{n}\right) \simeq-\frac{1}{\delta t} \int_{\boldsymbol{x}_{i-1 / 2}}^{\boldsymbol{x}_{i}}\left(\mathbf{U}_{i}^{n}-\mathbf{U}_{i-1 / 2}\left(t^{n+1}, x\right)\right) d x .
$$

We have used $\mathbf{U}_{i+1 / 2}(t, x)$ for the Riemann solver with the left state $\mathbf{U}_{i}^{n}$ and the right state $\mathbf{U}_{i+1}^{n}$. This Riemann solver is proposed in previous sub-sections and is based on the physical splitting of the characteristic waves. As the system (3.3) is non-conservative, the left-going and right-going fluctuations are not balanced.

\subsection{Multidimensional case}

In this sub-section we propose a multi-dimensional finite volume scheme based on the approximate Riemann solver proposed in the previous section. First, we decompose a computational domain into non-overlapping triangular and/or quadrilateral cells. Let us consider a cell $\mathcal{C}_{i}$ and denote the set of it's surrounding cells $\mathcal{C}_{j}$ by $\vartheta_{i j}$. We consider the decomposition of a cell $\mathcal{C}_{i}$ into non-overlapping sub-cells (triangles) such that each sub-cell contain an edge of the cell $\mathcal{C}_{i}$. Therefore each sub-cell of $\mathcal{C}_{i}$ also shares an edge with each surrounding cell $\mathcal{C}_{j}$. Compactly, this arrangement can be written as

$$
\mathcal{C}_{i}=\bigcup_{j \in \vartheta_{i j}} \mathcal{C}_{i j}
$$

We denote the normal vector at the interface $\partial \mathcal{C}_{i} \cap \partial \mathcal{C}_{j}=\partial \mathcal{C}_{i j} \cap \partial \mathcal{C}_{j}$ with the orientation from $\mathcal{C}_{i}$ to $\mathcal{C}_{j}$ by $\boldsymbol{n}_{i j}$. The situation is sketched in Fig. (1) for a cell $\mathcal{C}_{i}$ that is decomposed into nonoverlapping sub-cells $\mathcal{C}_{i j}$ by dotted lines. We denote the fluctuations at the interface $\partial \mathcal{C}_{i} \cap \partial \mathcal{C}_{j}$ by $\boldsymbol{\Psi}_{i j}\left(\mathbf{U}_{i}^{n}, \mathbf{U}_{j}^{n}\right)$ and $\Psi_{j i}\left(\mathbf{U}_{j}^{n}, \mathbf{U}_{i}^{n}\right)$.

Let $\mathcal{R}_{l r}(\boldsymbol{n}, t, \boldsymbol{x})$ be the solution of the approximate Riemann problem associated to left state $\mathbf{Q}_{l}(\boldsymbol{n})$ and right state $\mathbf{Q}_{r}(\boldsymbol{n})$. If the waves associated to the Riemann solvers at successive interfaces do not cross each other, we can use the Godunov-type scheme to define an updated solution:

$$
a_{i j} \mathbf{Q}_{i j}^{n+1}=\int_{\mathcal{C}_{i j}} \mathcal{R}_{i j}\left(\boldsymbol{n}_{i j}, t^{n+1}, \boldsymbol{x}\right) d \boldsymbol{x} \quad \text { where } \quad a_{i j}=\int_{\mathcal{C}_{i j}} d \boldsymbol{x}
$$

where $\mathbf{Q}_{i j}^{n+1} \equiv \mathbf{Q}_{i j}\left(\boldsymbol{x}_{i}, t^{n+1}\right)$. In fact $\mathbf{Q}_{i j}^{n+1}$ will be computed using the fluctuation splitting from the previous section. After updating the solution in the sub-cells, the mean solution is updated 
in each cell. The point is that, in the sub-cells the states are formulated in a local frame and this frame changes in each sub-cells. Then we use the Cartesian frame to represent the cell average. The vector of variables in this fixed Cartesian frame is:

$$
\mathbf{U}=\left(h, \quad h \boldsymbol{u}_{x}, \quad h \boldsymbol{u}_{y}, \quad \mathbb{P}_{x x}, \quad \mathbb{P}_{y y}, \quad \mathbb{P}_{x y}\right)^{\mathrm{T}}
$$

The transformation from the local to the Cartesian frame is simply denoted by $\mathbf{U}=\mathbf{U}(\mathbf{Q}(\boldsymbol{n}))$. Therefore the update in the cell is computed using:

$$
a_{i} \mathbf{U}_{i}^{n+1}=\sum_{j \in \vartheta_{i j}} a_{i j} \mathbf{U}\left(\mathbf{Q}_{i j}^{n+1}\right) \quad \text { where } \quad a_{i}=\int_{\mathcal{C}_{i}} d \boldsymbol{x}=\sum_{j \in \vartheta_{i}} a_{i j}
$$

Update of the local state $\mathbf{Q}_{i j}^{n+1}$ : In this context, the local fluctuations associated shear and acoustic sub-systems are computed. This is the analog of flux vector splitting (FVS) [2] applied to a non-conservative system. The two Riemann problems are independently solved with the same initial data with the states $\mathbf{Q}_{i}^{n}$ and $\mathbf{Q}_{j}^{n}$ to get the associated fluctuations as:

$$
\begin{aligned}
\boldsymbol{\psi}_{i j}^{a, n} & =\frac{1}{\delta t}\left(a_{i j} \mathbf{Q}_{i j}^{n}-\int_{\mathcal{C}_{i j}} \mathcal{R}_{i j}^{a}\left(\boldsymbol{n}_{i j}, t^{n+1}, \boldsymbol{x}\right) d \boldsymbol{x}\right) \\
\boldsymbol{\psi}_{i j}^{s, n} & =\frac{1}{\delta t}\left(a_{i j} \mathbf{Q}_{i j}^{n}-\int_{\mathcal{C}_{i j}} \mathcal{R}_{i j}^{s}\left(\boldsymbol{n}_{i j}, t^{n+1}, \boldsymbol{x}\right) d \boldsymbol{x}\right)
\end{aligned}
$$

with

$$
\boldsymbol{\psi}_{i j}^{n}=\boldsymbol{\psi}_{i j}^{a, n}+\boldsymbol{\psi}_{i j}^{s, n}
$$

and then the updated local state $\mathbf{Q}_{i j}^{n+1}$ is written as

$$
a_{i j} \mathbf{Q}_{i j}^{n+1}=a_{i j} \mathbf{Q}_{i j}^{n}-\delta t \sum_{j \in \vartheta_{i}} \boldsymbol{\psi}_{i j}^{n}
$$

Since the transformation $\mathbf{U}(\mathbf{Q})$ is linear, from Eq. (4.2), we obtain:

$$
a_{i} \frac{\mathbf{U}_{i}^{n+1}-\mathbf{U}_{i}^{n}}{\delta t}+\sum_{j \in \vartheta_{i}} \Psi_{i j}^{a}+\sum_{j \in \vartheta_{i}} \Psi_{i j}^{s}=0
$$

where $\boldsymbol{\Psi}_{i j}$ is the projection of the fluctuation $\boldsymbol{\psi}_{i j}$ into the Cartesian frame. The strategy written in the equation (4.7) enables one to develop a finite volume solver for unstructured grids.

\section{$5 \quad$ Numerical Tests}

We develop a cell-centered finite volume code that solves the SSWF model on 2D mixedunstructured grids. We apply equation (4.7) to solve non-dissipative part (3.1) and use 'standard splitting' approach [16] for purely dissipative part (2.4). The non-dissipative part is solved using the proposed physical splitting approach that involves solving Riemann problems associated with the shear and acoustic sub-systems. In the 'standard splitting' approach the numerical solution of the non-dissipative part is used as the initial condition to solve the purely dissipative part. Since the purely dissipative part is the system of ODEs, a suitable time-integration method can be used to solve it. We use the Euler's time-integration method to solve the purely dissipative part. 
An edge based algorithm is implemented for efficiency. For any edge $e$ in the grid, let $\mathcal{C}_{i}$ and $\mathcal{C}_{j}$ denote the two cells forming that edge. By $\boldsymbol{n}_{i j}$ we denote the normal vector to the edge $e$, pointing from cell $\mathcal{C}_{i}$ to $\mathcal{C}_{j}$. At any edge $e$, we define a local coordinate system $\left(\boldsymbol{n}_{i j}, \boldsymbol{n}_{i j}^{\perp}\right)$ and we transform the initial condition to this coordinate system. Then we solve the Riemann problems in this coordinate system. The time-step for the solver is computed as follows:

$$
\Delta t=\operatorname{CFL} \frac{\Delta h}{U_{c}}
$$

where $U_{c}$ is the maximum of the absolute value of the speed $\left(\|\boldsymbol{u}\|+\sqrt{g h+3 \mathbb{P}_{n n}}\right)$ and $\Delta h$ is the characteristic length. For structured grids it is specified as spacing $\Delta x$ in the $x$-direction while for unstructured grids it is specified as the length of the smallest edge in the grid. The Courant-Friedrichs-Lewy (CFL) number is used to control the time-step.

The algorithm to describe the solver can be summarized as:

1) Let $\boldsymbol{U}(t, x, y)$ be the solution at any time-instant $t$ and $\boldsymbol{Q}\left(t, n, n^{\perp}\right)$ be the solution into local coordinate system.

2) Using the solution $\boldsymbol{Q}\left(t, n, n^{\perp}\right)$ as the initial condition, we solve the approximate Riemann solver to calculate the partial fluctuations $\boldsymbol{\psi}_{i j}^{a}\left(\boldsymbol{n}_{i j}, \boldsymbol{Q}_{\boldsymbol{i}}, \boldsymbol{Q}_{\boldsymbol{j}}\right)$ associated to the acoustic subsystem.

3) Using the same initial condition $\boldsymbol{Q}\left(t, n, n^{\perp}\right)$, we solve the exact Riemann solver to calculate the partial fluctuations $\boldsymbol{\psi}_{i j}^{s}\left(\boldsymbol{n}_{i j}, \boldsymbol{Q}_{\boldsymbol{i}}, \boldsymbol{Q}_{\boldsymbol{j}}\right)$ associated to the shear subsystem.

4) Using Godunov-type scheme written in the equation (4.7), we update the solution to the next time step $\left(\boldsymbol{Q}\left(t+\Delta t, n, n^{\perp}\right)\right)$.

5) Then we transform back this updated solution to the Cartesian coordinate system $(\boldsymbol{U}(t+$ $\Delta t, x, y))$.

6) If a problem contains dissipative terms, we use the updated solution $\boldsymbol{U}(t+\Delta t, x, y)$ as the initial condition and apply Euler's time-integration method to solve the purely dissipative part of the model.

7) We repeat above steps for the next time-step.

Next we present results of some 1D and 2D numerical tests to validate the solver based on the proposed fluctuation splitting scheme.

\subsection{Convergence}

On the account of the availability of an exact solution to the system (3.1) [7], convergence of the solver is studied on different types of grids. The exact solution is written as:

$$
\begin{gathered}
h=\frac{h_{0}}{1+\beta^{2} t^{2}} \\
\boldsymbol{u}=\frac{\beta}{1+\beta^{2} t^{2}}\left(\begin{array}{c}
\beta t x+y \\
\beta t y-x
\end{array}\right) \\
\mathbb{P}=\frac{1}{\left(1+\beta^{2} t^{2}\right)^{2}}\left(\begin{array}{cc}
\lambda+\gamma \beta^{2} t^{2} & (\lambda-\gamma) \beta t \\
(\lambda-\gamma) \beta t & \gamma+\lambda \beta^{2} t^{2}
\end{array}\right)
\end{gathered}
$$


The exact solution with $h_{0}=1 \mathrm{~m}, \lambda=0.1 \mathrm{~m}^{2} / \mathrm{s}^{2}, \gamma=0.01 \mathrm{~m}^{2} / \mathrm{s}^{2}$ and $\beta=0.001 \mathrm{~s}^{-1}$ is used to specify initial and boundary conditions. The square domain of size $L_{x}=L_{y}=10 \mathrm{~m}$ is used to generate different types of structured and unstructured grids. As expected, the first order convergence is observed in all cases. The details of the convergence tests are summarized below:

1) A series of structured quadrangular grids is generated with 40, 80, 160 and 320 number of points on the each boundary of the domain. With CFL $=0.4$, the $L_{1}$ and $L_{2}$-norm of the errors $(E)$ in each variable are plotted versus mesh size $\Delta h$ on log-log scale at the final time $t=10 \mathrm{~s}$, in Fig. (2).

2) A series of structured triangular grids is generated with 40, 80, 160 and 320 number of points on the each boundary of the domain. With CFL $=0.2$, the $L_{1}$ and $L_{2}$-norm of the errors $(E)$ in each variable are plotted versus mesh size $\Delta h$ on log-log scale at the final time $t=10 \mathrm{~s}$, in Fig. (3).

3) A series of unstructured quadrangular grids is generated with 20, 40, 80 and 160 number of points on the each boundary of the domain. With CFL $=0.4$, the $L_{1}$ and $L_{2}$-norm of the errors $(E)$ in each variable are plotted versus mesh size $\Delta h$ on log-log scale at the final time $t=10 \mathrm{~s}$, in Fig. (4).

4) A series of unstructured triangular grids is generated with 20, 40, 80 and 160 number of points on the each boundary of the domain. With $\mathrm{CFL}=0.2$, the $L_{1}$ and $L_{2}$-norm of the errors $(E)$ in each variable are plotted versus mesh size $\Delta h$ on log-log scale at the final time $t=10 \mathrm{~s}$, in Fig. (5).

\section{$5.21 \mathrm{D}$ shear test}

For 1D shear test, we use the domain of unit length with the initial condition as $h=0.01 \mathrm{~m}$, $u=0, \mathbb{P}_{x x}=\mathbb{P}_{y y}=10^{-4} \mathrm{~m}^{2} / \mathrm{s}^{2}$ and $\mathbb{P}_{x y}=0 \mathrm{~m}^{2} / \mathrm{s}^{2}$. The discontinuity in the initial condition positioned in the variable $v$ at $x=0.5 \mathrm{~m}$. To the left of the discontinuity $v=0.2 \mathrm{~m} / \mathrm{s}$ and to the right $v=-0.2 \mathrm{~m} / \mathrm{s}$. The computations are performed with $\mathrm{CFL}=0.5$. Frames in Fig. (6) show $v, \mathbb{P}_{x x}$ and $\mathbb{P}_{y y}$ at $t=10 s$ computed on different grids with 100,500 and 10000 grid cells. In this figure, the convergence of the solution can be clearly seen.

\section{$5.31 \mathrm{D}$ dam break test}

For $1 \mathrm{D}$ dam break case, once again we use a domain of unit length with the initial discontinuity located at $x=0.5 \mathrm{~m}$. The depth $h$ is $0.02 \mathrm{~m}$ to the left and $0.01 \mathrm{~m}$ to the right of the discontinuity. The velocity components $u$ and $v$ are initialized to $0 \mathrm{~m} / \mathrm{s}$ and the components of the stress tensor are $\mathbb{P}_{x x}=\mathbb{P}_{y y}=10^{-4} \mathrm{~m}^{2} / \mathrm{s}^{2}$ and $\mathbb{P}_{x y}=0 \mathrm{~m}^{2} / \mathrm{s}^{2}$. The computations are performed with $\mathrm{CFL}=$ 0.5. Frames in Fig. (7) show $h, u$ and $\mathbb{P}_{x x}$ at the time $t=0.5 \mathrm{~s}$ computed on different grids with 100, 500 and 10000 grid cells. In this figure, the convergence of the solution can be clearly seen.

\section{$5.41 \mathrm{D}$ roll waves}

For this test problem, the bottom topography is an inclined plane with an angle $\theta$ in the $x$ direction

$$
\nabla \mathrm{b}=\left(\begin{array}{c}
-\tan \theta \\
0
\end{array}\right)
$$


Dissipative contributions and initial conditions are such that the flow starts from a perturbed equilibrium and develops into so called roll waves $[3,5,10]$. The initial condition is specified as

$$
\begin{array}{r}
h(x, y, 0)=h_{0}\left(1+a \sin \left(\frac{2 \pi x}{L_{x}}\right)\right), \quad u(x, y, 0)=\sqrt{\frac{g h_{0} \tan \theta}{C_{f}}}, \quad v(x, y, 0)=0 \\
\mathbb{P}_{x x}(x, y, 0)=\mathbb{P}_{y y}(x, y, 0)=\frac{\phi h^{2}(x, y, 0)}{2}, \quad \mathbb{P}_{x y}(x, y, 0)=0
\end{array}
$$

where, $\theta$ (inclination angle $)=0.05011, C_{f}($ Chèzy coefficient $)=0.0036, h_{0}=7.98 \times 10^{-3}, a$ (amplitude of perturbation) $=0.05, \phi=22.76 \mathrm{~s}^{-2}, C r=0.00035, L x=1.3 \mathrm{~m}$. These parameters are taken from the tests performed in [9] that correspond to the Brock's experiments [3, 4]. Periodic boundary condition is used in the $x$-direction. In the $y$-direction, $v$ and $\mathbb{P}_{x y}$ are specified to zero and Neumann boundary condition is specified on the other variables. The time-step is computed by specifying CFL $=0.8$ and 1000 grid cells are used. Figure (8) shows the difference between the absolute value of numerical $h$ profiles obtained by Euler's and classical fourth order Runge-Kutta $(R K 4)$ time-integration method for the purely dissipative model (2.4). The difference is plotted at $t=25 \mathrm{~s}$ of the simulation and it can be seen that the difference is not very significant. Henceforth, we use the Euler's time-integration method for the purely dissipative part of the model.

Numerically obtained roll wave profile is compared with the Brock's [3, 4] experimental data in Fig. (9). Numerical results show a good agreement with the experimental data. Three distinct regions of the roll wave profile as observed in Brock's experiment: a steep wave front, a zone with progressive increment in the depth and a hydraulic jump are clearly seen in the Fig. (9). The enstrophy $\Psi$ that plays the roll of the mathematical entropy increases sharply across the shocks which can be seen in Fig. (10).

\section{$5.52 \mathrm{D}$ roll waves}

In this test case, similar initial and boundary conditions are specified as 1D roll waves except that the flow is perturbed in both the directions :

$$
h(x, y, 0)=h_{0}\left(1+a\left(\sin \frac{2 \pi m x}{L_{x}}+\sin \frac{2 \pi k y}{L_{y}}\right)\right)
$$

where $L_{x}=1.3 \mathrm{~m}$ and $L_{y}=0.5 \mathrm{~m}$. The initial condition is specified by $m=1$ and $k=1$ in all the computations presented here. This $2 \mathrm{D}$ test corresponds to the similar test from [7] where authors report the formation of transverse waves in their computations. Similar transverse waves are obtained in the computations on structured quadrangular grids. Slightly different waves are observed on truly unstructured grids, whereas structured triangular grids are found to suppress the onset/formation of transverse waves owing to the numerical dissipation added by them.

Figures (11), (12) and (13) show the results obtained by the computations performed on structured quadrangular grid with $1000 \times 400$ points and $\mathrm{CFL}=0.4$. The value of the initial $\Delta t$ is $3.7345 \times 10^{-04}$. In Fig. (11), the depth $(h)$ is plotted at $t=57.8 \mathrm{~s}$ showing stable transverse structures which are similar to those obtained in [7]. Frames in the Fig. (12) show top view of the variables $h, \mathbb{P}_{x x}, u$ and $v$ plotted at $t=57.8 s$ showing the effect of transverse waves in each variable. Frames in Fig. (13) show formation of stable transverse structures. At $t=17.09 \mathrm{~s}$ $1 \mathrm{D}$ roll wave profile with a shock is observed, followed by the onset of transverse waves. At $t=19.95 \mathrm{~s}$ waves originating at the shock and near boundaries can be seen. Further, these waves start appearing in the domain as shown at $t=27.78 \mathrm{~s}$. Finally, a stable transverse structure appears as shown in the Fig. (13) at $t=44.73$. 
The time instant at which the onset of transverse waves occurs depends upon the value of CFL and hence time-step. These transverse wave structures appears to be an instability phenomenon in the SSWF model triggered by the numerical noise acting as perturbations. A series of computations is performed on the structured quadrangular grid with $1000 \times 400$ points with different values of $\mathrm{CFL}=1,0.9,0.8$ and 0.7 . Hence the values of CFL used here are close to the numerical stability limit $(\mathrm{CFL}=1)$. The time-step $(\Delta t)$ is plotted verses time $(t)$ in Fig. (14) for each simulation. Dynamics in each simulation is accompanied by two decrements in $\Delta t$ which can be seen in each curve of Fig. (14). The first of which corresponds to the formation of a quasi-1D roll wave profile, nearly at the time $(t=5 \mathrm{~s})$. The interval of the stability of this quasi-1D wave seems to larger when CFL used is smaller. In such cases the second and sharp decrease in the curve of $\Delta t$ appears latter, delaying the appearance of transverse structures. This can be viewed as the system containing transverse waves which are superimposed on the quasi-1D profiles. Depending on the numerical noise (which is larger near the stability limit) transverse waves can be observed earlier. Another feature which can be noted from $\Delta t$ curves is that, as CFL decreases fluctuations in the time-steps decrease. For the simulations with higher CFL, unsteady nature of transverse waves cause such fluctuations in the time-step. For small values of CFL stable waves are seen, as shown in Fig. (11), (12) and (13). The results presented above are computed on a fine grid, however similar results are obtained for the coarse grids like the one with $300 \times 50$ points.

For simulations on structured triangular grids, two types of grids are used as shown in frames of Fig. (15). The grid shown in the top frame of Fig. (15) is generated by splitting all the rectangles in the same orientation while the grid shown in the bottom frame of Fig. (15) is generated by splitting all rectangles in alternate orientations. The results shown here are computed on the grids with $520 \times 200$ points $(206562$ cells) each. The value of CFL is specified as 0.4 for both simulations which corresponds to the initial times step $7.4648 \times 10^{-04}$. With the first type of grid, one transverse structure is observed near the boundary which is shown in the top frame of the Fig. (15). This single structure appears roughly at $t=25 \mathrm{~s}$ but the transverse structures are not observed further. The shape of this single structure looks similar to each structure obtained in the simulations with structured quadrangular grids. With the second type of grid, stable 1D roll waves profile is observed without any transverse waves which is shown in the bottom frame of Fig. (15). Cells in these two types of grids are orientated in specific directions with respect to the flow adding numerical dissipation and hence suppressing the transverse waves.

As expected, transverse structures obtained in the simulations on truly unstructured grids are affected by the lack of alignment of cells with respect to the flow direction and do not have specific shape. This can be attributed to the numerical dissipation provided by the truly unstructured grids in all directions. It can be viewed as the real situations where disturbances in the flow are isotropic. The top frame in Fig. (16) shows the zoomed snapshot of the unstructured quadrangular grid with 173251 cells and the plot of $h$ at $t=40 \mathrm{~s}$. The CFL is specified as 0.4 in this computation which account to the initial $\Delta t=2.634 E-04$. Similarly, the bottom frame in the Fig. (16) shows the zoomed snapshot of the unstructured triangular grid with 172898 cells and the plot of $h$ at $t=40 \mathrm{~s}$. The CFL is specified as 0.4 in this computation which accounts to the initial $\Delta t=3.6201 E-04$. The transverse waves obtained in these simulations does not have any specific shape but they appear realistic. Such transverse structures with no specific shapes were observed in the field experiments in [10].

\section{Conclusion}

We have proposed a fluctuation splitting finite volume method for a non-conservative model of SSWF. This model was proposed in $[14,7]$ and the numerical scheme based on the directional splitting was proposed in the [7]. This directional splitting method is tricky to apply on un- 
structured grids. In this work, we use physical splitting to separate the characteristic waves of the model equations and form the shear and acoustic sub-systems. An exact Riemann solver is developed for the shear sub-system and an approximate Riemann solver is developed for the acoustic sub-system. A Godunov-type scheme is proposed that uses partial fluctuations obtained from each Riemann solvers. The fact that these Riemann solvers are formulated into a local coordinate system allows us to develop a finite volume code for mixed unstructured grids. In [7] equation of energy conservation has been solved along with the SSWF model (2.1), whereas in this paper only system (2.1) has been solved. This certainly gives an advantage on the cost of computations.

Some numerical tests have been performed to validate the proposed scheme. As expected, the first order convergence is obtained for this scheme. A good agreement with experimental data is seen for $1 \mathrm{D}$ roll wave test case. In $2 \mathrm{D}$ roll wave simulations, transverse waves are observed which are qualitatively similar to those obtained in [7]. These transverse waves occur as an instability phenomenon of the SSWF model triggered by the numerical errors. Onset and stability of transverse waves depend upon the numerical noise while the shape of transverse structures depends on the nature of the grid. Several numerical results obtained with structured as well as unstructured grids are presented to demonstrate the applicability of the solver.

\section{References}

[1] D. Balsara, J. Li, and G. Montecinos. An efficient, second order accurate, universal generalized riemann problem solver based on the hlli riemann solver. submitted, 2018.

[2] D. Balsara, Gino I. Montecinos, and E. Toro. Exploring various flux vector splittings for the magnetohydrodynamic system. Journal of Computational Physics, 311:1 - 21, 2016.

[3] R. Brock. The development of roll-waves trains in open channels. J. Hydraulics Division, 95:1401 - 1428, 1969.

[4] R. Brock. Periodic permanent roll waves. J. Hydraulics Division, 96:2565 - 2580, 1970.

[5] Alexander Chesnokov, Valery Liapidevskii, and Irina Stepanova. Roll waves structure in two-layer hele-shaw flows. Wave Motion, 73:1 - 10, 2017.

[6] M. Dumbser and D. Balsara. A new efficient formulation of the hllem riemann solver for general conservative and non-conservative hyperbolic systems. Journal of Computational Physics, 304:275 - 319, 2016.

[7] S. Gavrilyuk, K. Ivanova, and N. Favrie. Multi-dimensional shear shallow water flows: Problems and solutions. Journal of Computational Physics, 366:252 - 280, 2018.

[8] S.K. Godunov. A difference scheme for numerical computation of discontinous solutions of equations of fluids dynamics. Math. Sb., 47:271-290, 1959.

[9] K. Ivanova, S. Gavrilyuk, B. Nkonga, and G. Richard. Formation and coarsening of rollwaves in shear shallow water flows down an inclined rectangular channel. Computers and Fluids, 159:189 - 203, 2017.

[10] X. Leng and H. Chanson. Breaking bore : Physical observation of roller characteristics. Mechanics Research Communications, 65:24 - 29, 2015.

[11] G. Dal Maso, P. LeFloch, and F. Murat. Definition and weak stability of a non conservative product. J. Math. Pures Appl., 74:483-548, 1995. 
[12] M. L Munoz-Ruiz and C. Pares. Godunov method for nonconservative hyperbolic systems. ESAIM: Mathematical Modelling and Numerical Analysis, 41(1):169-185, 2007.

[13] G. Richard and S. Gavrilyuk. The classical hydraulic jump in a model of shear shallow-water flows. Journal of Fluid Mechanics, 725:492 - 521, 2013.

[14] V. M. Teshukov. Gas dynamic analogy for vortex free-boundary flows. Journal of Applied Mechanics and Technical Physics, 48:303-309, 2007.

[15] S.A. Tokareva and E.F. Toro. A flux splitting method for the baer-nunziato equations of compressible two-phase flow. Journal of Computational Physics, 323:45 - 74, 2016.

[16] E. Toro. Shock-capturing methods for free-surface shear flows. Wiley, 2001.

[17] E.F. Toro and M.E. Vazquez-Cendon. Flux splitting schemes for the euler equations. Computers and Fluids, 70:1 - 12, 2012.

[18] Eleuterio F. Toro, Cristobal E. Castro, and Bok Jik Lee. A novel numerical flux for the 3d euler equations with general equation of state. Journal of Computational Physics, 303:80 94, 2015. 


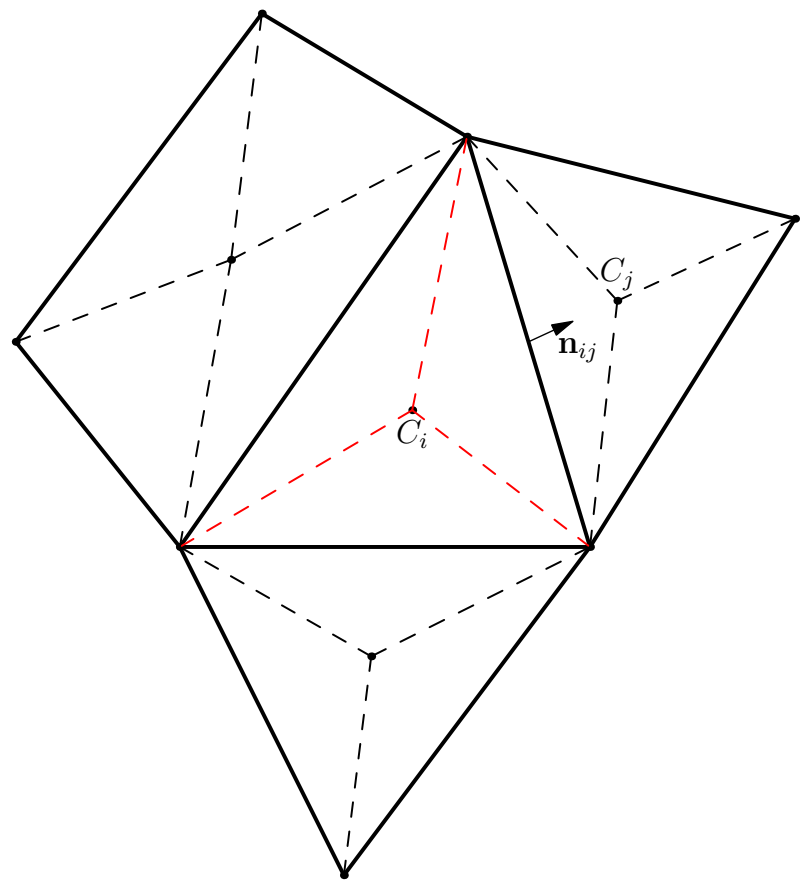

FIGURE 1. Sketch showing sub-cells formed in a cell $\mathcal{C}_{i}$. Each sub-cell in the cell $\mathcal{C}_{i}$ shares an edge with cells $\mathcal{C}_{j}$ (cells surrounding a cell $\mathcal{C}_{i}$ ). The normal vector $\boldsymbol{n}_{\boldsymbol{i j}}$ to the edge directs from the cell $\mathcal{C}_{i}$ to $\mathcal{C}_{j}$ 

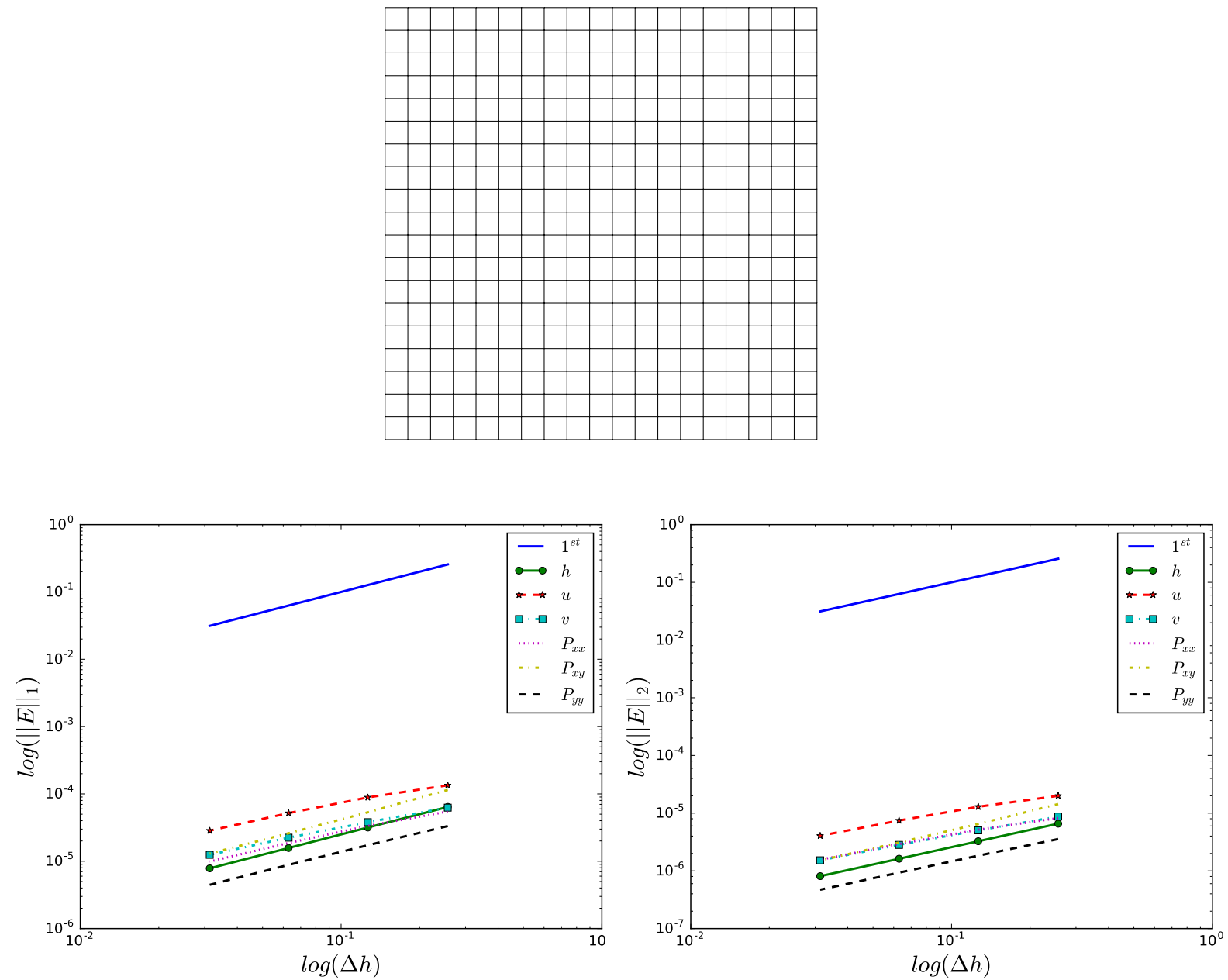

Figure 2. Structured quadrangular mesh : $L_{1}$ and $L_{2}$-norm of the errors $(E)$ plotted versus mesh size $(\Delta h)$ plotted on log-log scale at $t=10 \mathrm{~s}$. 

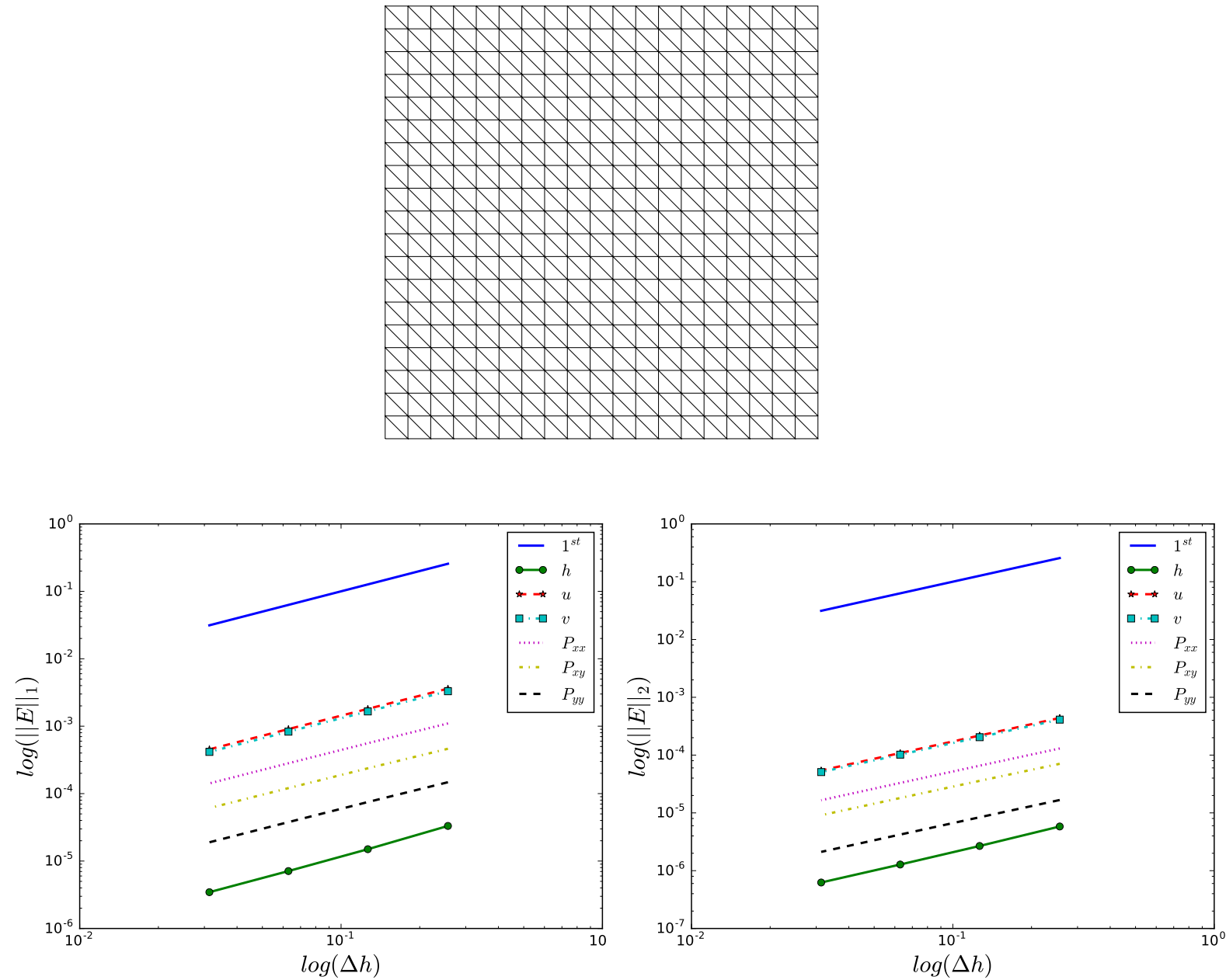

Figure 3. Structured (quasi) triangular mesh : $L_{1}$ and $L_{2}$-norm of the errors $(E)$ plotted versus mesh size $(\Delta h)$ plotted on log-log scale at $t=10 \mathrm{~s}$. 

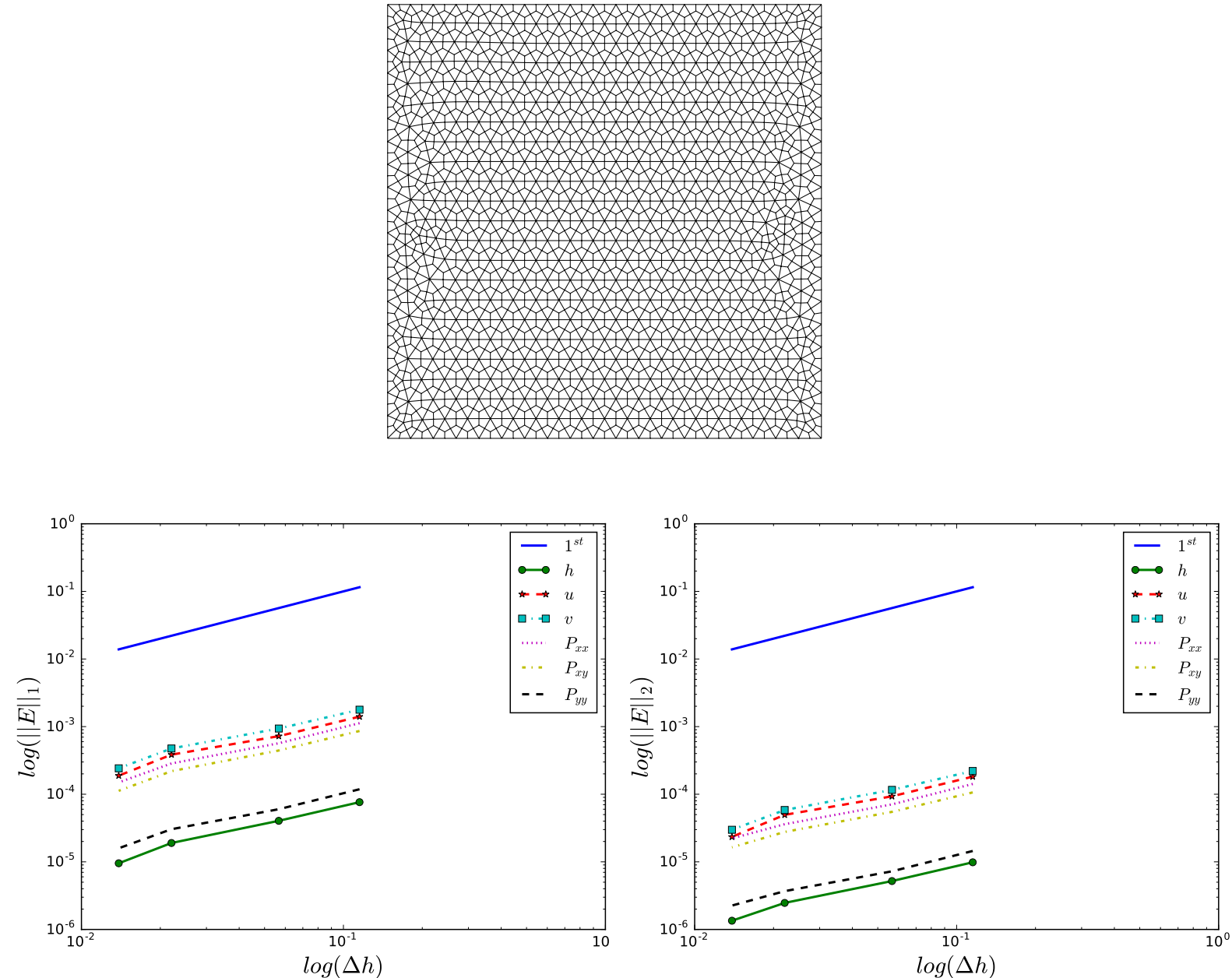

FiguRE 4. Unstructured quadrangular mesh : $L_{1}$ and $L_{2}$-norm of the errors $(E)$ plotted versus mesh size $(\Delta h)$ plotted on log-log scale at $t=10 \mathrm{~s}$. 

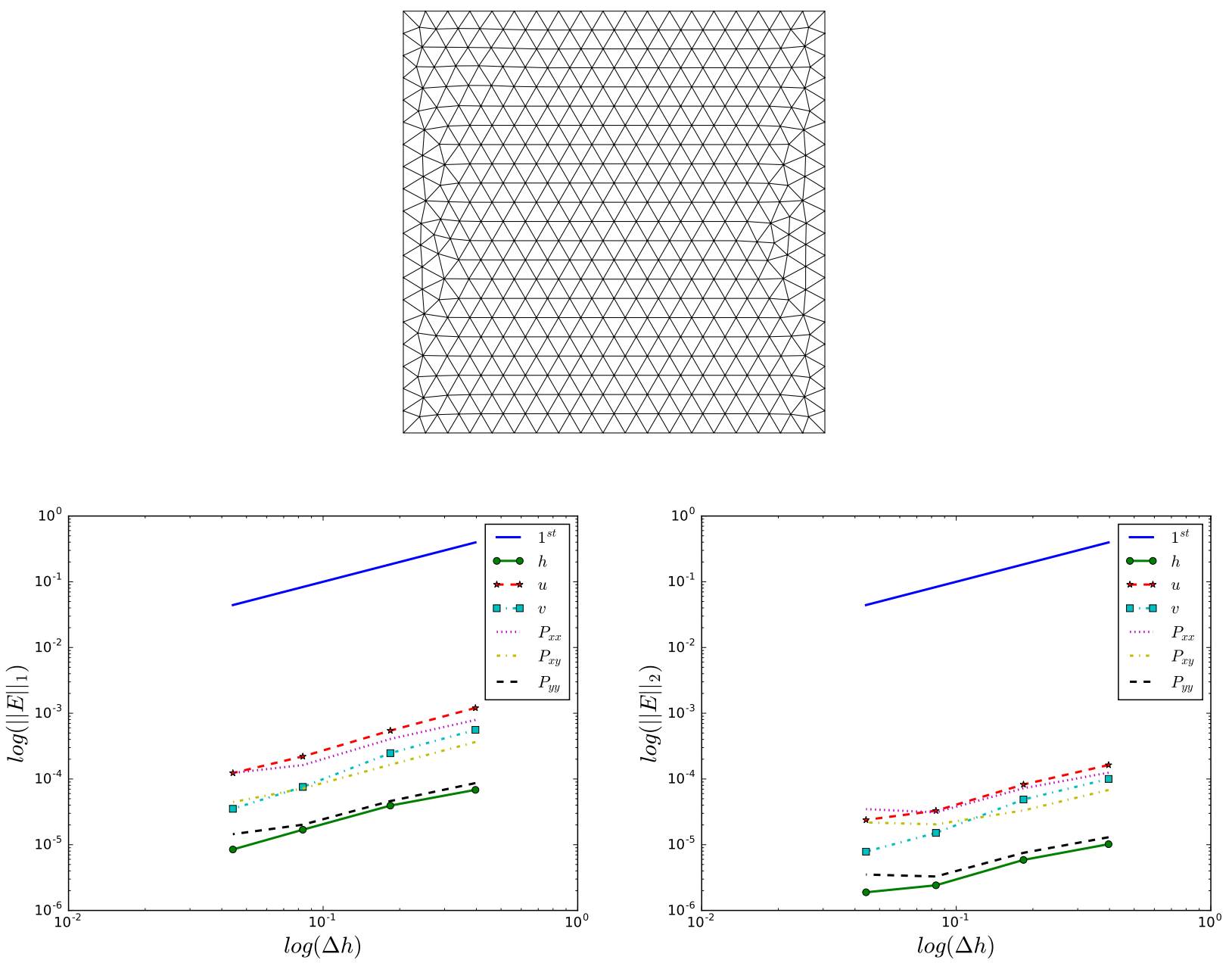

Figure 5. Unstructured triangular mesh : $L_{1}$ and $L_{2}$-norm of the errors $(E)$ plotted versus mesh size $(\Delta h)$ plotted on log-log scale at $t=10 \mathrm{~s}$. 

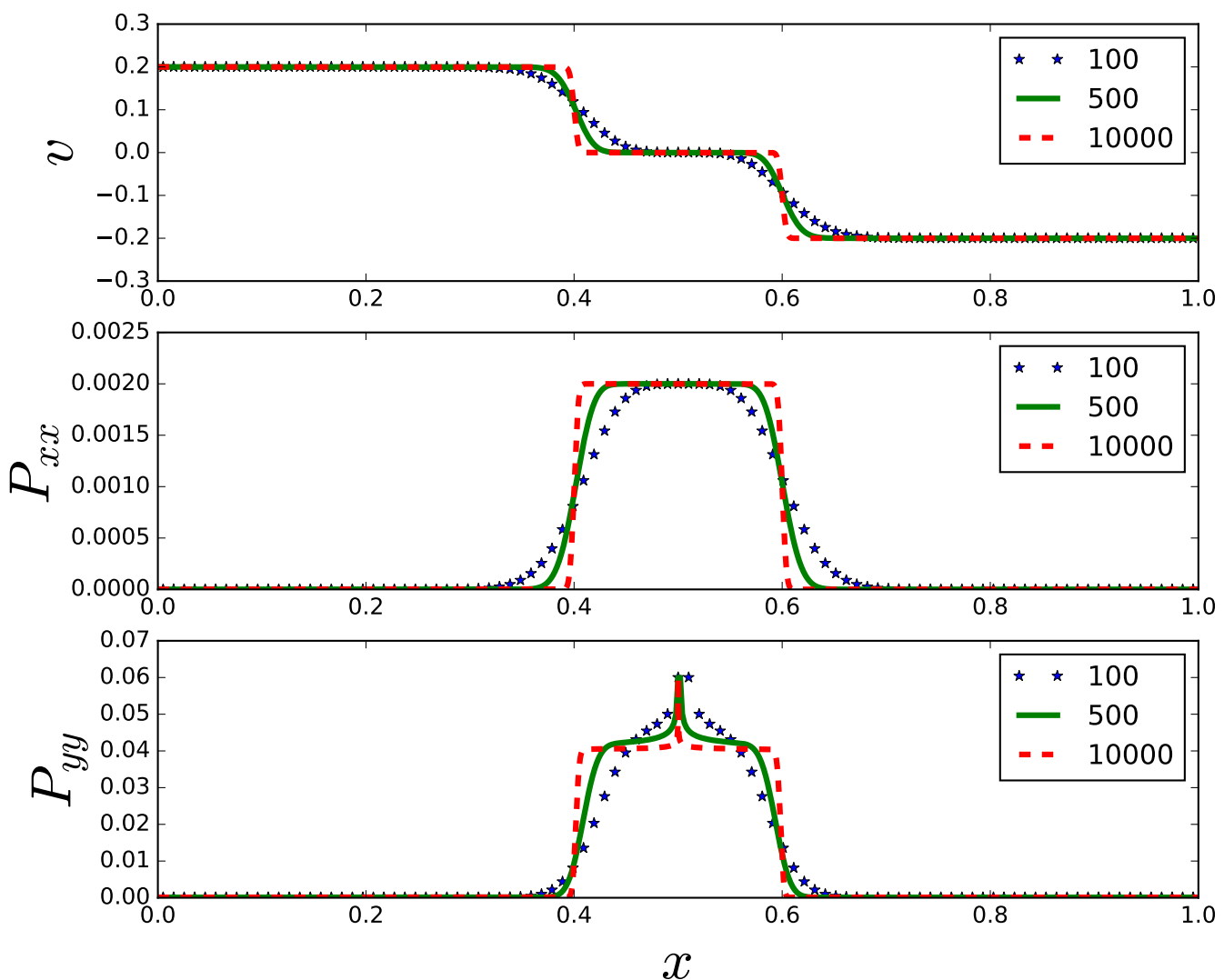

Figure 6. Numerical solution of $1 \mathrm{D}$ shear test showing $v, \mathbb{P}_{x x}$ and $\mathbb{P}_{y y}$ on the different grids with 100 , 500 and 10000 grid cells. The CFL is specified as 0.5. 

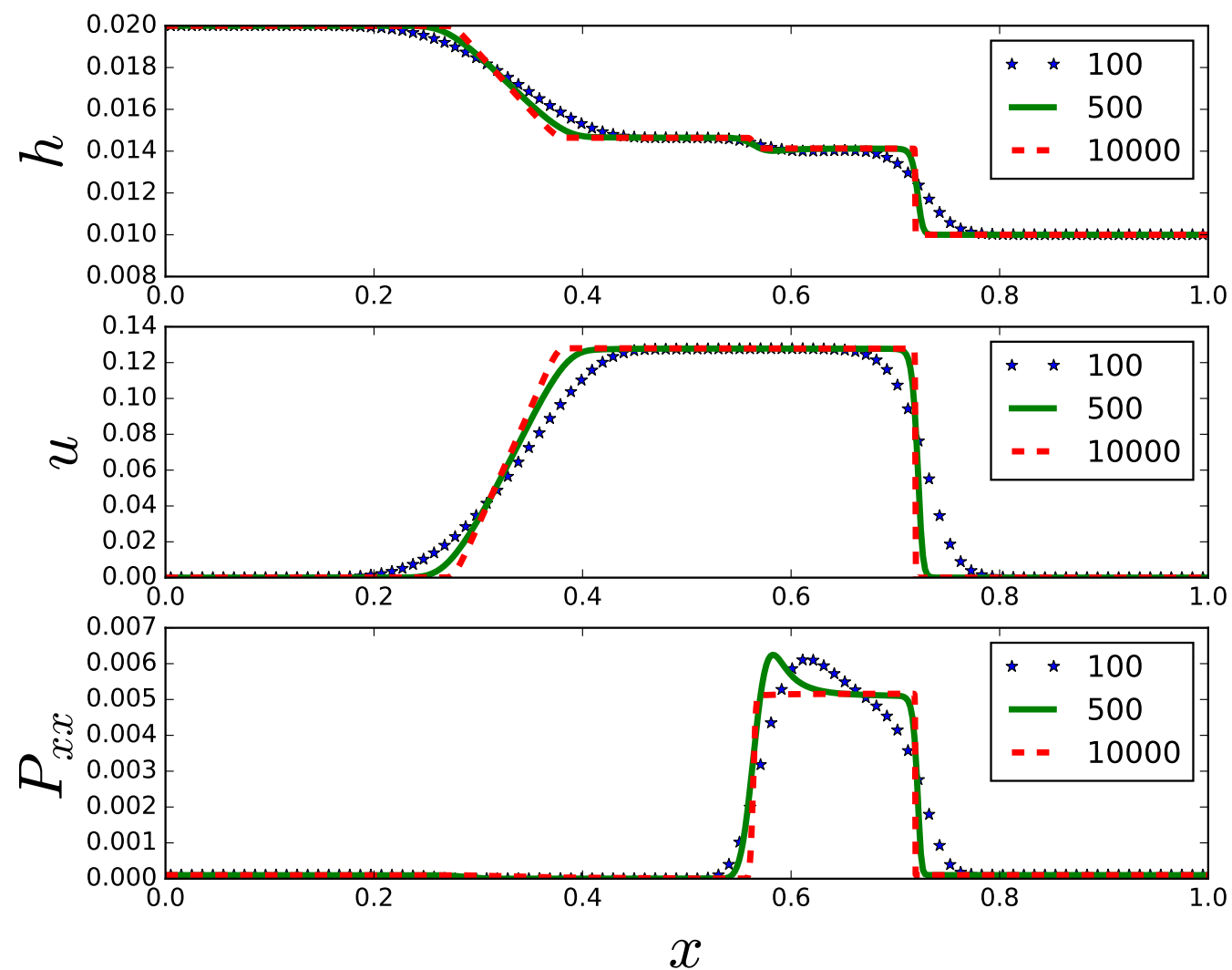

Figure 7. Numerical solution of $1 \mathrm{D}$ dam-break test showing $h, u$ and $\mathbb{P}_{x x}$ on the different grids with 100, 500 and 10000 grid cells. The CFL is specified as 0.8 . 


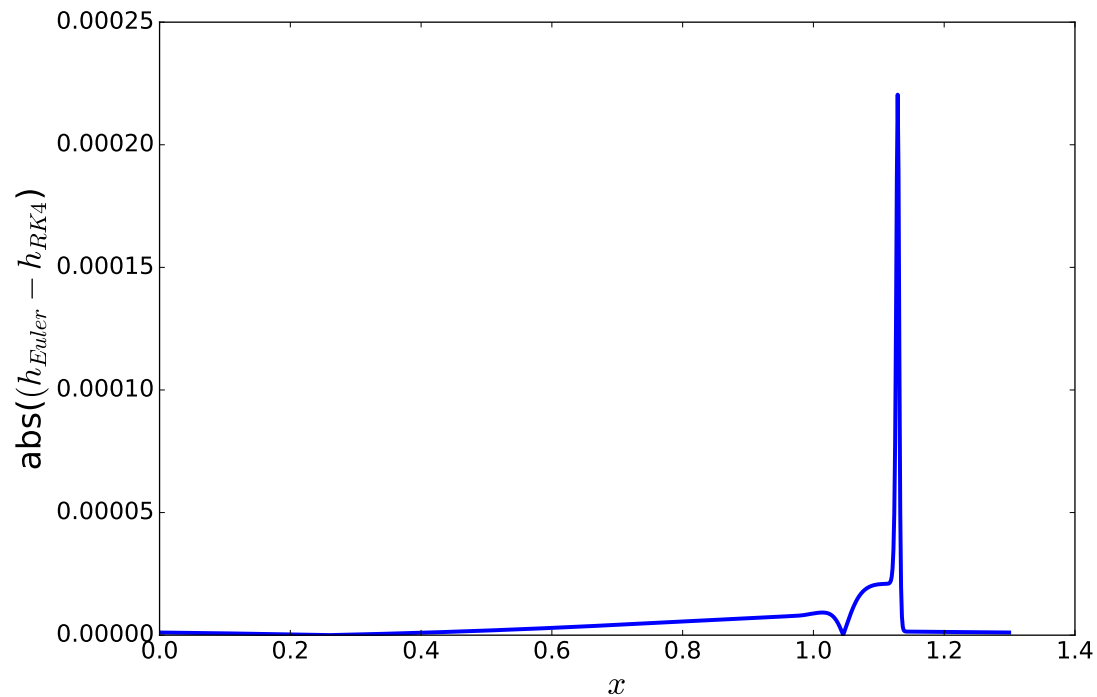

Figure 8. Absolute of the difference between $h$-profiles obtained by Euler's and $R K 4$ time-integration for the purely dissipative model. Both computations are performed using 1000 grid cells and CFL $=0.8$. The plot is shown for the solution at time $t=25 \mathrm{~s}$.

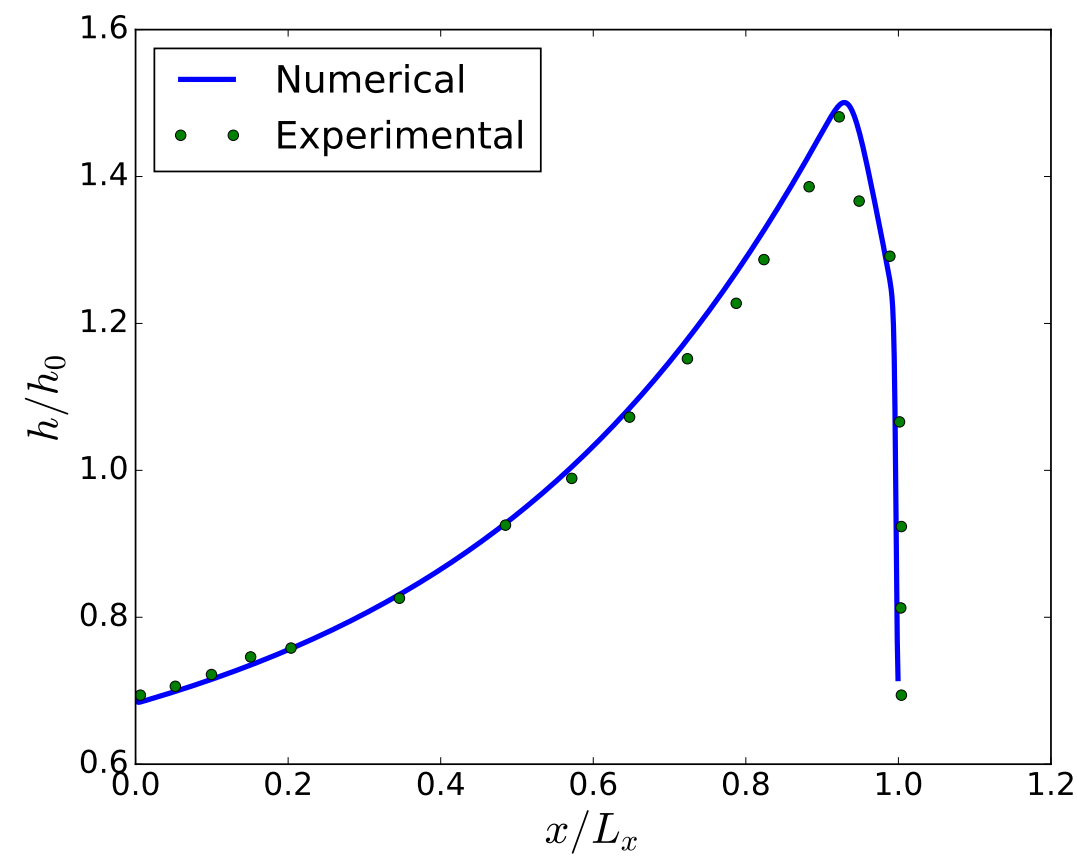

Figure 9. Comparison of numerically obtained 1D roll wave profile for the depth and the Brock's experimental data. The numerical profile shown here is obtained with 1000 grid cells and CFL $=0.8$ at the time instant $t=25.11 \mathrm{~s}$. 

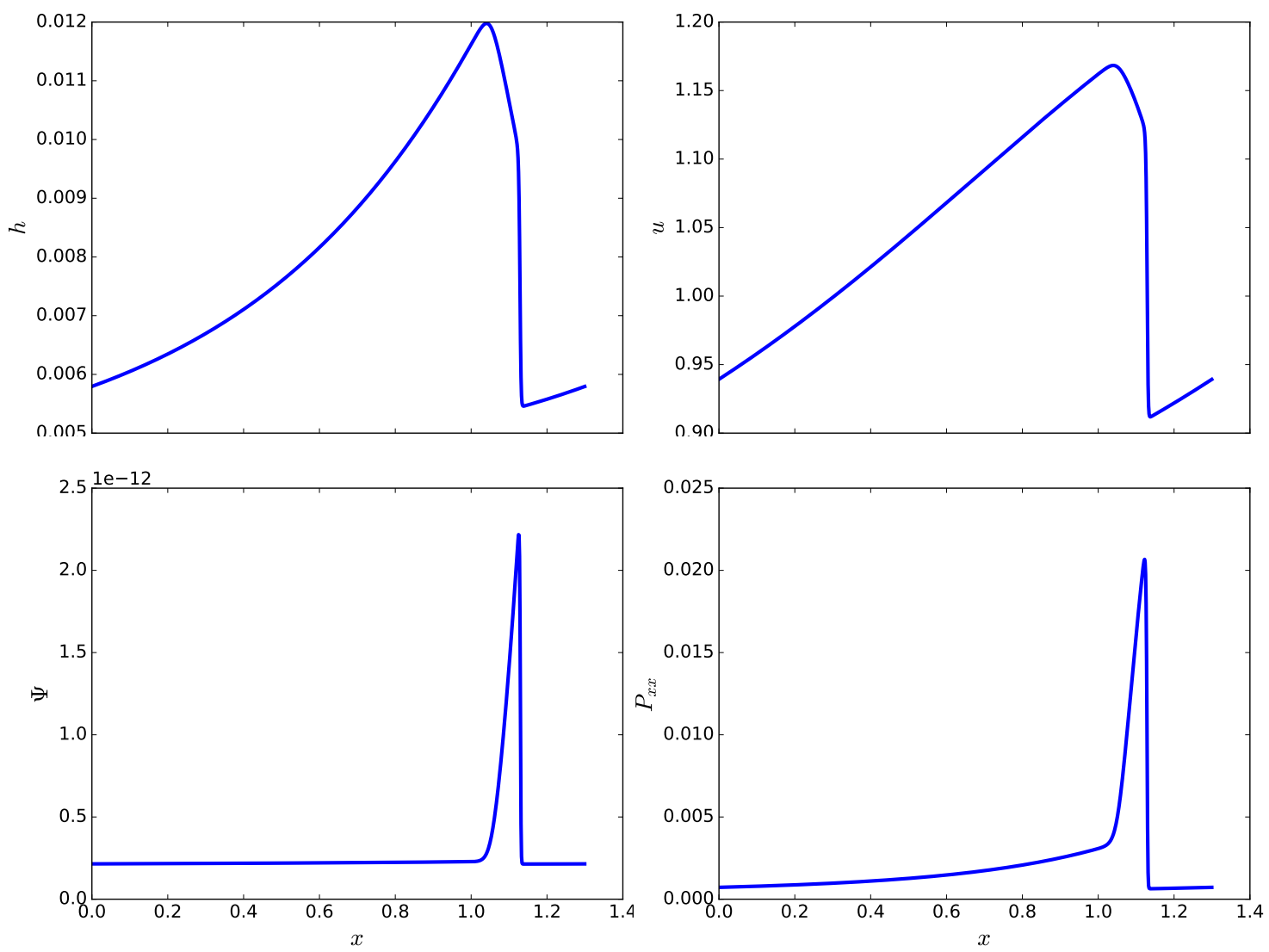

Figure 10. Numerical solution for 1D Roll wave problem at the time instant $t=25 \mathrm{~s}$ with 1000 grid cells and $\mathrm{CFL}=0.8$.

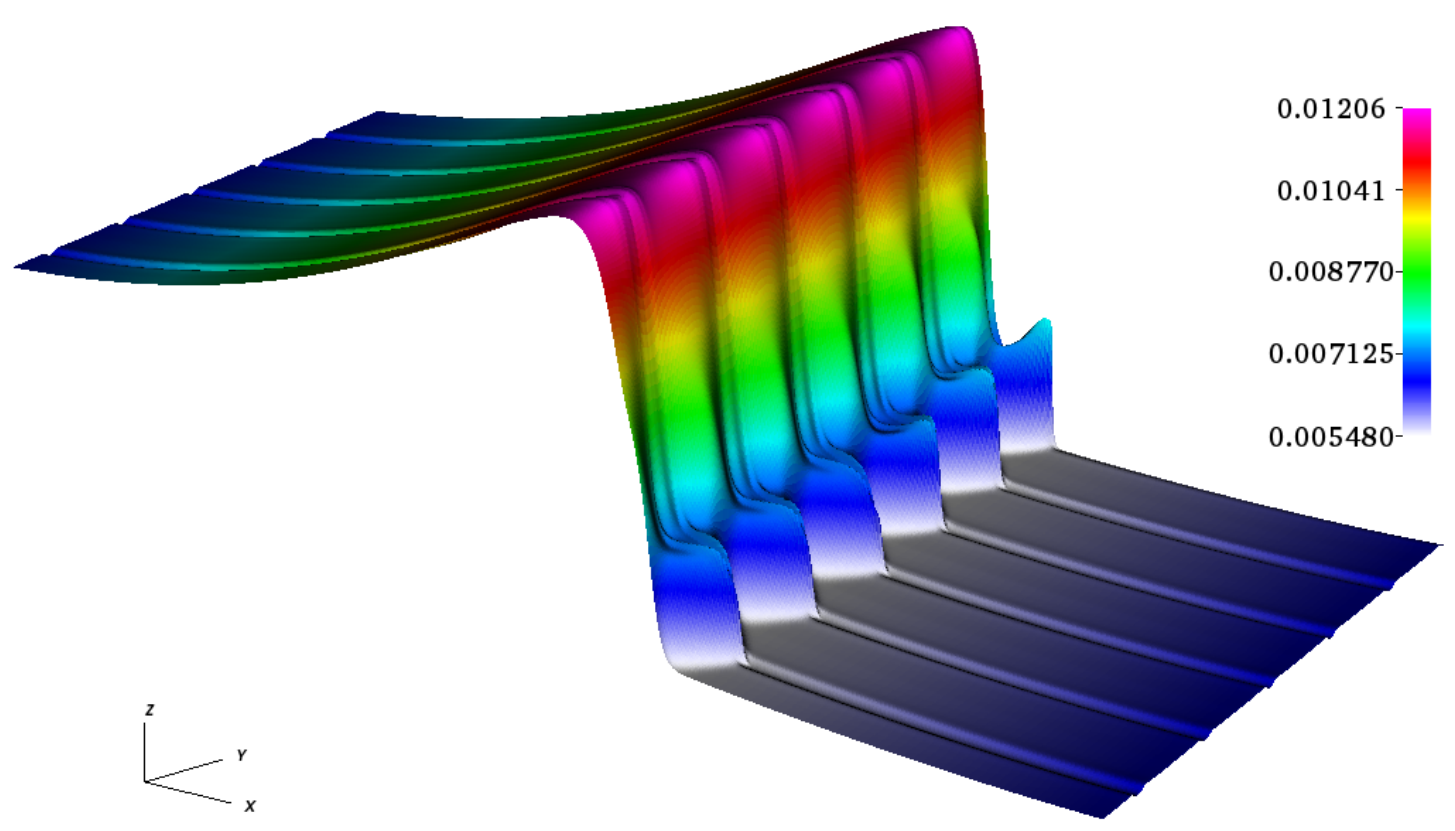

Figure 11. 2D roll waves: The depth $(h)$ profile showing stable transverse structures at the time $t=57.8 \mathrm{~s}$ on structured quadrangular grid with $1000 \times 400$ points and $\mathrm{CFL}=0.4$. 

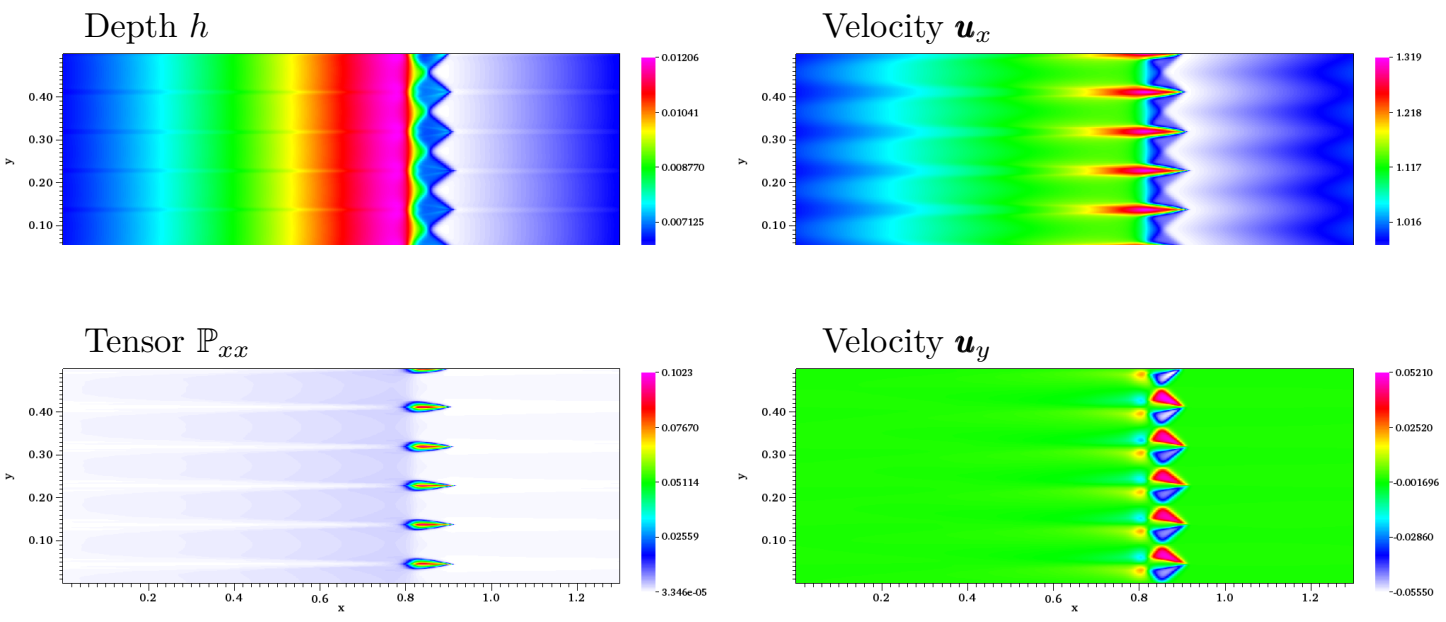

Figure 12. 2D roll waves: Top view of $2 \mathrm{D}$ roll waves with stable transverse structures at the time $t=57.8 \mathrm{~s}$ on structured quadrangular grid with $1000 \times 400$ points and $\mathrm{CFL}=0.4$.
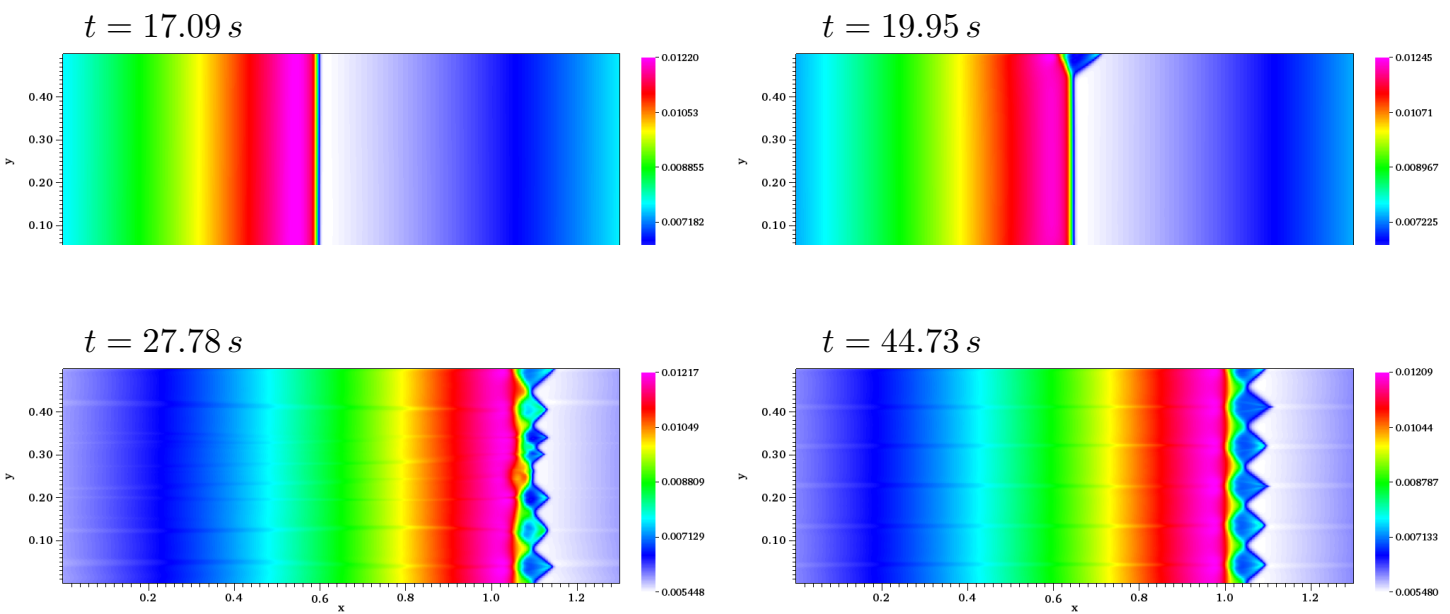

Figure 13. 2D roll waves : Evolution of stable transverse structures on structured quadrangular grid with $1000 \times 400$ points and $\mathrm{CFL}=0.4$. 


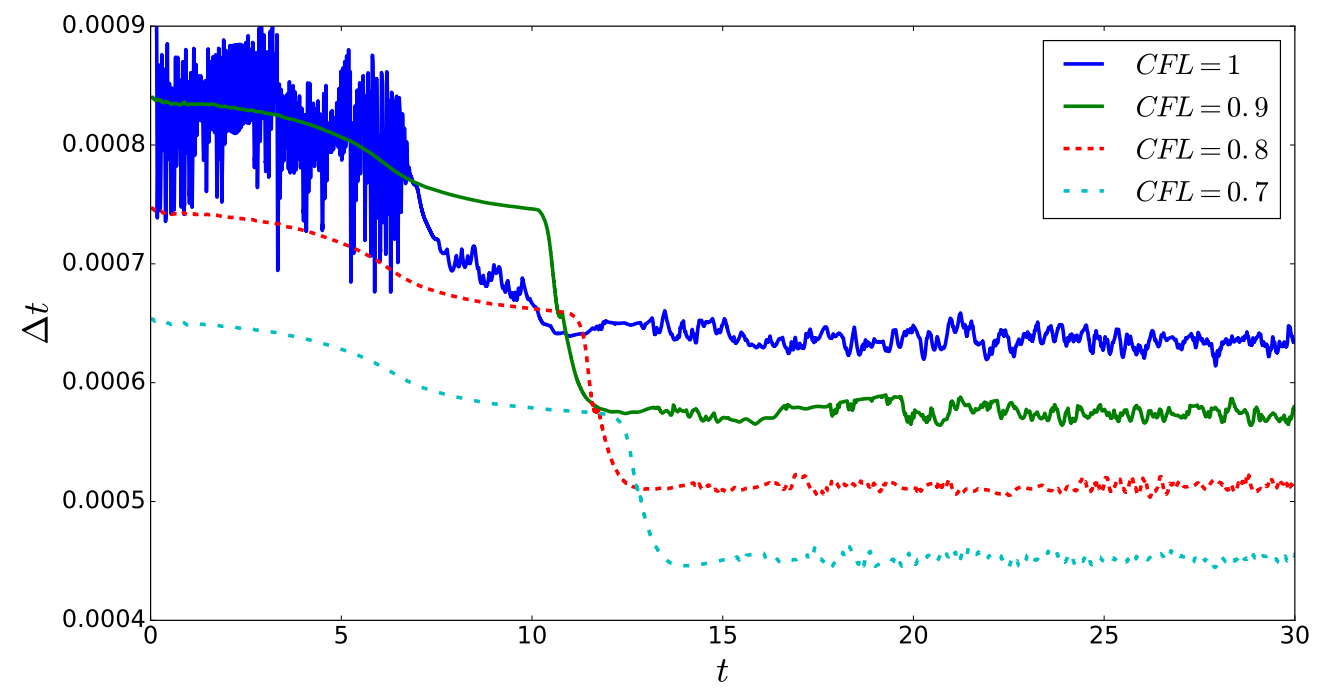

Figure 14. 2D roll waves: The curves of time-steps $\Delta t$ plotted versus time $t$ for the simulations of $2 \mathrm{D}$ roll waves on structured quadrangular grid with $1000 \times 400$ points. Sharp decrease in each curve denote the onset of transverse waves. 

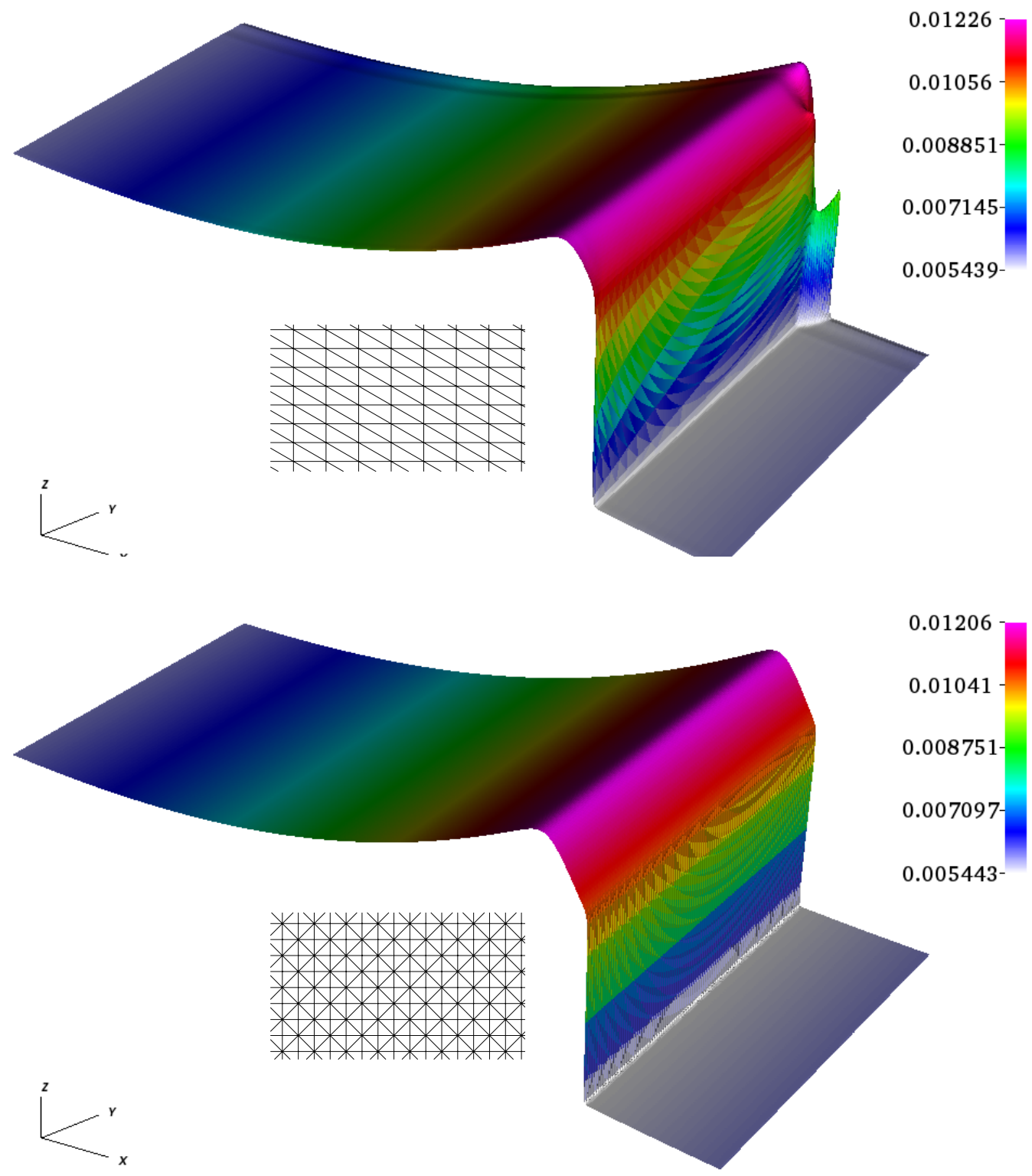

Figure 15. 2D roll waves: The depth $(h)$ profile at time $t=40 \mathrm{~s}$ obtained on structured triangular grids with 206562 cells and $\mathrm{CFL}=0.4$. Top and bottom frames show results obtained on the grid with rectangles split in same and alternate orientation, respectively. 

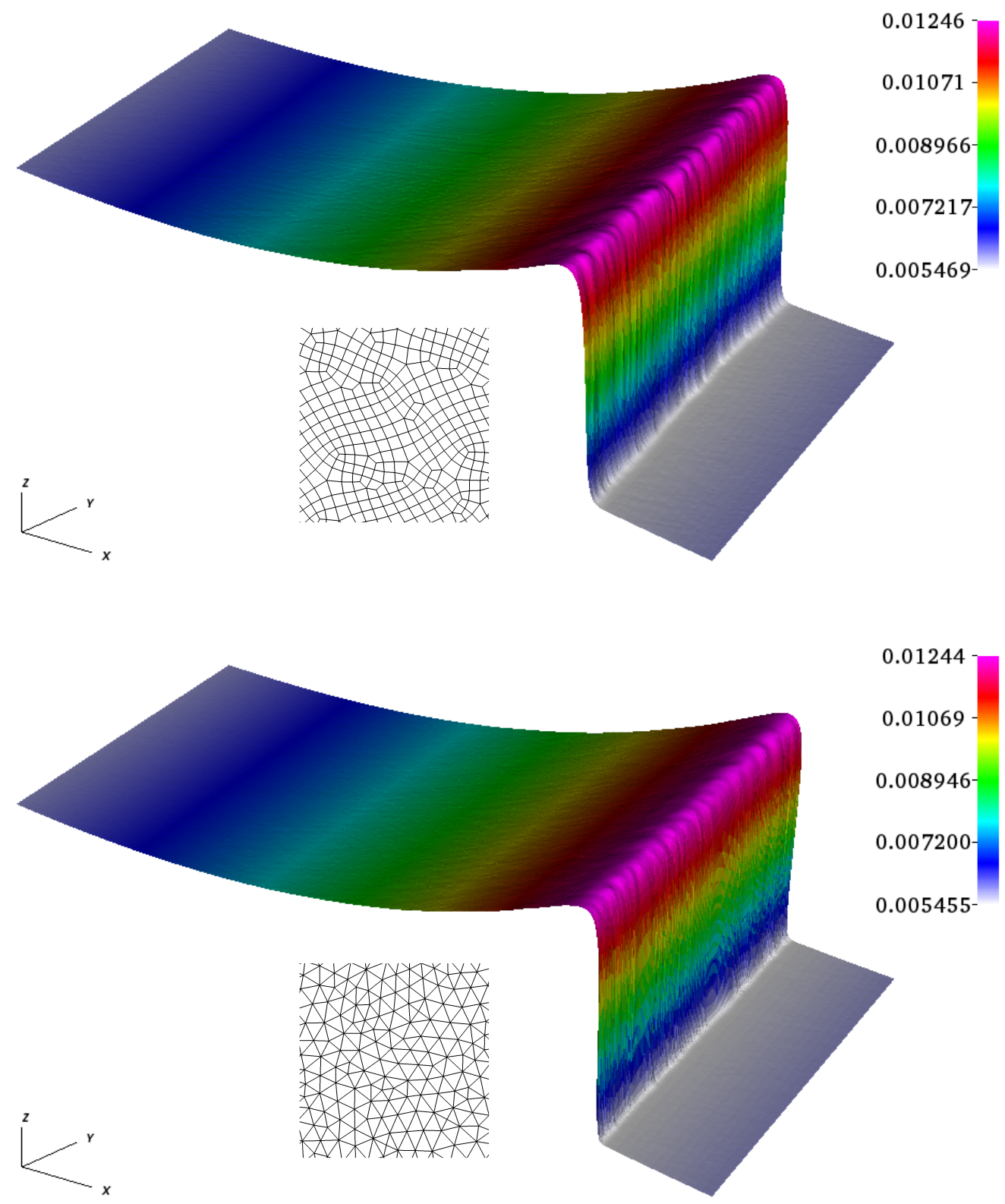

Figure 16. 2D roll waves: The depth $(h)$ profile at time $t=40 \mathrm{~s}$ obtained with $\mathrm{CFL}=0.4$ on the unstructured quadrangular grid with 173251 cells (top) and on the unstructured triangular grid with 172898 cells (bottom). 